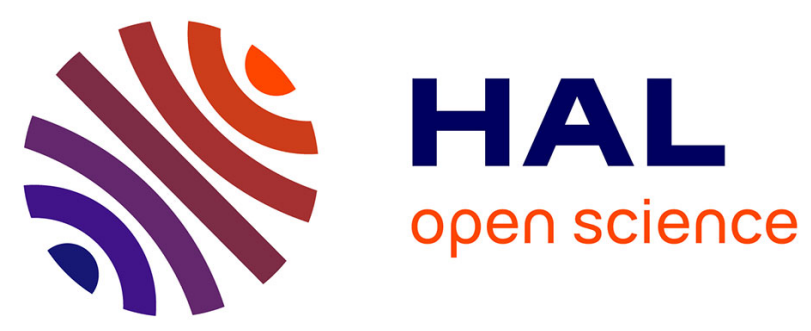

\title{
Analysis of a finite volume method for a cross-diffusion model in population dynamics
}

\author{
Boris Andreianov, Mostafa Bendahmane, Ricardo Ruiz Baier
}

\section{To cite this version:}

Boris Andreianov, Mostafa Bendahmane, Ricardo Ruiz Baier. Analysis of a finite volume method for a cross-diffusion model in population dynamics. Mathematical Models and Methods in Applied Sciences, 2011, 21 (02), pp.307-344. 10.1142/S0218202511005064 . hal-00458737v2

\section{HAL Id: hal-00458737 \\ https://hal.science/hal-00458737v2}

Submitted on 1 Mar 2010

HAL is a multi-disciplinary open access archive for the deposit and dissemination of scientific research documents, whether they are published or not. The documents may come from teaching and research institutions in France or abroad, or from public or private research centers.
L'archive ouverte pluridisciplinaire HAL, est destinée au dépôt et à la diffusion de documents scientifiques de niveau recherche, publiés ou non, émanant des établissements d'enseignement et de recherche français ou étrangers, des laboratoires publics ou privés.

\section{다)(1) $(5$}

Distributed under a Creative Commons Attribution - NonCommerciall 4.0 International 


\title{
ANALYSIS OF A FINITE VOLUME METHOD FOR A CROSS-DIFFUSION MODEL IN POPULATION DYNAMICS
}

\author{
BORIS ANDREIANOV ${ }^{\dagger}$, MOSTAFA BENDAHMANE $^{\star}$, AND RICARDO RUIZ BAIER ${ }^{\ddagger}$
}

\begin{abstract}
The main goal of this work is to propose a convergent finite volume method for a reaction-diffusion system with cross-diffusion. First, we sketch an existence proof for a class of cross-diffusion systems. Then standard two-point finite volume fluxes are used in combination with a nonlinear positivity-preserving approximation of the cross-diffusion coefficients. Existence and uniqueness of the approximate solution are addressed, and it is also shown that the scheme converges to the corresponding weak solution for the studied model. Furthermore, we provide a stability analysis to study pattern-formation phenomena, and we perform two-dimensional numerical examples which exhibit formation of nonuniform spatial patterns. From the simulations it is also found that experimental rates of convergence are slightly below second order. The convergence proof uses two ingredients of interest for various applications, namely the discrete Sobolev embedding inequalities with general boundary conditions and a space-time $L^{1}$ compactness argument that mimics the compactness lemma due to S.N. Kruzhkov. The proofs of these results are given in the Appendix.
\end{abstract}

\section{IntRoduction}

1.1. Scope and presentation of the problem. The interaction between species has been widely studied with reaction-diffusion models. Species may interact both by affecting each other's demography and/or by influencing each others dispersal patterns. If one considers that most organisms are neither simple diffusers nor move always randomly, but can display preferential directions of movement 2], then the underlying model could include a cross-diffusion term, which will allow to take into account the population pressures induced by competing species. Shigesada and Kawasaki 44 examined the invasion process in a Lotka-Volterra type competition with diffusion. In the studied phenomenon, the entire domain is initially occupied by the resident species, and a few individuals of the invading species arrive. The spread of the invading species turns out to be a traveling wave and it is known that depending on the parameters of the model, the invading species either completely displaces the resident, or the system reaches a coexistent value. It is also known that, under certain conditions, if the invading species has only a slight competitive advantage over the resident species, the displacement process will be slower. In a related context, Okubo et al. 41 investigated the invasion process of grey squirrels (Sciurus carolinensis) after their introduction in Britain, which displaced the red squirrel (Sciurus vulgaris). A two-species Lotka-Volterra reaction-diffusion competition system modeling the interaction between these squirrels was proposed by Shigesada, Kawasaki and Teramoto in [45] (the well known SKT system).

Date: Received: October 28, 2009

Updated: March 1, 2010.

1991 Mathematics Subject Classification. 65M60, 35K55, 92D25.

Key words and phrases. Cross-diffusion, finite volume approximation, convergence to the weak solution, patternformation

†Département de Mathématiques, Université de Franche-Comté, 16 route de Gray, 25030 Besançon Cedex, France. Email: boris.andreianov@univ-fcomte.fr.

*Institut de Mathématiques de Bordeaux UMR CNRS 5251, Université Victor Segalen Bordeaux 2, F-33076 Bordeaux Cedex, France.

Email: mostafa_bendahmane@yahoo.fr.

${ }_{\ddagger}$ Modeling and Scientific Computing, CMCS-MATHICSE-SB, Ecole Polytechnique Fédérale de Lausanne EPFL, Station 8, CH-1015, Lausanne, Switzerland.

Email: ricardo.ruiz@epfl.ch. 
Of particular interest for us is the following strongly coupled nonlinear cross-diffusion system

$$
\begin{gathered}
\partial_{t} u-D_{1} \Delta u-\operatorname{div}\left(\mathcal{A}_{11}(u, v) \nabla u+\mathcal{A}_{12}(u, v) \nabla v\right)=u\left(a_{1}-b_{1} u-c_{1} v\right), \\
\partial_{t} v-D_{2} \Delta v-\operatorname{div}\left(\mathcal{A}_{21}(u, v) \nabla u+\mathcal{A}_{22}(u, v) \nabla v\right)=v\left(a_{2}-b_{2} u-c_{2} v\right), \quad(x, t) \in Q_{T}:=\Omega \times(0, T], \\
\frac{\partial u}{\partial \eta}=\frac{\partial v}{\partial \eta}=0, u(x, 0)=u_{0}(x), \quad v(x, 0)=v_{0}(x),
\end{gathered}
$$

where $\mathcal{A}=\left(\mathcal{A}_{i j}\right)_{1 \leq i, j \leq 2}$ is the cross-diffusion matrix that we take equal to

$$
\mathcal{A}(u, v)=\left(\begin{array}{cc}
u+v & u \\
v & u+v
\end{array}\right) .
$$

Here $\Omega \subset \mathbb{R}^{l}, l=2$ or $l=3$, is a bounded open domain with piecewise smooth boundary $\partial \Omega$. The quantities of interest $u(x, t), v(x, t)$ denote population densities in $(x, t)$ of red and grey squirrels (resident and invading species respectively). The change in distribution range is caused only by self diffusion, cross-diffusion, and growth. In addition $a_{1}, a_{2}$ are the Malthusian growth coefficients, and $b_{1}, c_{2}$ and $b_{2}, c_{1}$ are the coefficients of intra- and inter-species competition, respectively. We consider that the diffusive constants $D_{1}, D_{2}$ are positive. It is convenient, although not necessary, to assume that the intra-species competition coefficients $b_{1}, c_{2}$ are also strictly positive. Written in matrix form with the unknown $\mathbf{u}:=(u, v)$ (understood as a column vector), 1.1) reads

$$
\partial_{t} \mathbf{u}-\operatorname{div}\left[\left(\begin{array}{cc}
D_{1} & 0 \\
0 & D_{2}
\end{array}\right) \nabla \mathbf{u}\right]-\operatorname{div}[\mathcal{A}(\mathbf{u}) \nabla \mathbf{u}]=\left(\begin{array}{c}
F(\mathbf{u}) \\
G(\mathbf{u})
\end{array}\right),
$$

where the reaction terms are given by

$$
F(u, v):=u\left(a_{1}-b_{1} u-c_{1} v\right), \quad G(u, v):=v\left(a_{2}-b_{2} u-c_{2} v\right) .
$$

A simpler model herein considered is given by the following Lotka-Volterra two species competition model with self-diffusion [39, 41]:

$$
\begin{aligned}
\partial_{t} u-D_{1} \Delta u & =u\left(a_{1}-b_{1} u-c_{1} v\right), \\
\partial_{t} v-D_{2} \Delta v & =v\left(a_{2}-b_{2} u-c_{2} v\right) .
\end{aligned}
$$

Such system is known to possess global smooth non-negative solution. Moreover, as the classical Lotka-Volterra differential equations, it is known 40, that (1.4) uniformly oscillates around a nontrivial, neutrally stable equilibrium point. Recent studies dealing with systems of this type also include [7, 12, 32, 37, 42, 43, (the list of references is far from being complete).

The more involved system $(1.1),(1.2)$ has received a considerable attention as well. Many works have been proposed to investigate the conditions for existence and uniqueness of weak/global solutions, and stability/instability issues. Recent results include for instance [15], where the method of "additional generating conditions" is used to find exact solutions to the SKT model in the one-dimensional case. In [16, 33, 38, 46] precise conditions are given for the existence of unstable equilibrium points for SKT-type systems, other works include analysis of weak solutions of cross-diffusion systems [9], global existence and uniform boundedness of solution 47. From the numerical analysis viewpoint, the list of references is drastically narrowed. In [26, 27] the authors prove the convergence of a semidiscretization in time scheme in one space dimension by introducing a transformation of the unknowns. Several results in one spatial dimension using a deterministic particle method are presented in [28. In [6], the authors propose a fully discrete finite element scheme for a cross-diffusion system using an entropy inequality, and also present one-dimensional examples. Using a finite difference scheme, global existence of a weak solution is obtained in [13, and the global existence of a SKT-type model is established in 14 via a positivity-preserving Euler-Galerkin method. Our contribution aims to present a finite volume scheme to obtain approximate solutions to the SKT model $(1.1),(1.2)$. These numerical 
approximations are shown to converge to the corresponding weak solutions, and we provide twodimensional numerical examples illustrating the accuracy and performance of the scheme. Moreover, we report on the formation of nonuniform spatial patterns following the analysis presented in [46.

The method we apply works if the diffusion matrix $\mathcal{A}(\mathbf{u})$ in 1.2 is replaced by a general matrix

$$
\mathcal{A}(u, v)=\left(\begin{array}{ll}
\mathcal{A}_{11} & \mathcal{A}_{12} \\
\mathcal{A}_{21} & \mathcal{A}_{22}
\end{array}\right)
$$

satisfying the assumptions

$$
\begin{gathered}
\forall u, v \geq 0 \quad \mathcal{A}_{12}(0, v)=0, \text { and } \mathcal{A}_{21}(u, 0)=0, \\
\forall u, v \geq 0 \quad \forall \mathbf{w} \in \mathbb{R}^{2} \quad(\mathcal{A}(u, v) \mathbf{w}, \mathbf{w}) \geq \frac{1}{C}\|\mathcal{A}(u, v)\|\|\mathbf{w}\|^{2},
\end{gathered}
$$

where $(\cdot, \cdot)$ is the usual scalar product on $\mathbb{R}^{2}$, and

$$
\forall u, v \geq 0 \quad\|\mathcal{A}(u, v)\| \leq C\left(1+u^{r}+v^{r}\right) \quad \text { with } r<\left\{\begin{aligned}
4, & \text { if } l=2 \\
10 / 3, & \text { if } l=3 .
\end{aligned}\right.
$$

Notice that 1.6 entails

$$
\forall u, v \geq 0 \quad \mathcal{A}_{11}(u, v) \geq 0 \text { and } \mathcal{A}_{22}(u, v) \geq 0 .
$$

Assumptions 1.8, 1.5 allow for nonnegative solutions; assumption (1.6) expresses the positivity of the cross-diffusion matrix; and (1.7) is a kind of growth assumption on $\mathcal{A}$. Clearly, 1.2 satisfies the above assumptions.

The analysis of this paper is restricted to the case of positive cross-diffusion matrices $\mathcal{A}$. This case is sometimes referred to as "weak cross-diffusion". It has been shown by Chen and Jüngel [13] that a class of cross-diffusion systems 1 with matrices $\mathcal{A}$ generalizing 1.2 possesses an entropy that allows to establish existence without the positivity assumption on $\mathcal{A}$. The numerical approximation of such "strong cross-diffusion" problems will be the subject of a forthcoming paper.

1.2. Outline of the paper. The remaining part of this paper is organized as follows. In Section 2 we define weak solutions to system (1.1), (1.2), and briefly discuss the existence issue. In Section 3 we introduce some notations for the finite volume method, and we present our numerical scheme and the main theorem of convergence. The proof of this convergence result is divided into Section 4 (basic a priori estimates and existence of a solution to the scheme), Section 5 (compactness for discrete solutions) and Section 6 (convergence to a weak solution). Notice that the result of Section 5 is based upon a discrete analogue of the Kruzhkov $L^{1}$ compactness lemma (cf. [34]); the statement and the proof of the Lemma are postponed to Appendix A. This compactness result is of interest for a wide variety of finite volume approximations of evolution equations; therefore Appendix A contains the essential notation and it can be read independently of the rest of the paper. Similarly, Appendix B is independent of the rest of the paper; it contains a proof (adapted from the idea of Eymard, Gallouët and Herbin 24]) of the discrete Sobolev embedding inequalities for the case of Neumann or mixed boundary conditions. Finally, in Section 7 we present numerical examples in two space dimensions, putting into evidence the good performance of the scheme, and we provide the corresponding error histories. In Section 9 we outline a stability-instability analysis to deduce sufficient conditions for spatial patterns to appear.

\section{WEAK SOLUTIONS}

Definition 2.1. A pair $\mathbf{u}=(u, v)$ of functions is a weak solution of 1.1 if $u, v \in L^{2}\left(0, T ; H^{1}(\Omega)\right)$, and the following identities hold for all test functions $\varphi, \xi \in \mathcal{D}([0, T) \times \Omega)$ :

$$
-\iint_{\Omega_{T}} u \partial_{t} \varphi d x d t+D_{1} \iint_{\Omega_{T}} \nabla u \cdot \nabla \varphi d x d t+\iint_{\Omega_{T}}\left[\mathcal{A}_{11}(u, v) \nabla u+\mathcal{A}_{12}(u, v) \nabla v\right] \cdot \nabla \varphi d x d t
$$




$$
\begin{gathered}
=\iint_{\Omega_{T}} F(u, v) \varphi d x d t+\int_{\Omega} u_{0}(x) \varphi(0, x) d x, \\
-\iint_{\Omega_{T}} v \partial_{t} \xi d x d t+D_{2} \iint_{\Omega_{T}} \nabla v \cdot \nabla \xi d x d t+\iint_{\Omega_{T}}\left[\mathcal{A}_{21}(u, v) \nabla u+\mathcal{A}_{22}(u, v) \nabla v\right] \cdot \nabla \xi d x d t \\
=\iint_{\Omega_{T}} G(u, v) \xi d x d t+\int_{\Omega} v_{0}(x) \xi(0, x) d x .
\end{gathered}
$$

In model (1.1), we are only interested in non-negative solutions, i.e. in $(u, v)$ such that $u \geq 0$, $v \geq 0$ a.e. on $Q_{T}$. In fact, existence of a weak non-negative solution for system (1.1) will be shown by proving convergence of our numerical scheme; notice that, at least in the case of the cross-diffusion 1.2), existence can be proved directly (see [8, 9, 10] for details). Here we sketch a somewhat simpler convergence proof, which is the one mimicked in Sections 4, 5,6 .

Theorem 2.2. Assume that the cross-diffusion matrix $\mathcal{A}$ fulfills the requirements $(1.5)-(1.7)$. Then for all $u_{0}, v_{0} \in L^{2}(\Omega)$ there exists a non-negative weak solution of 1.1 .

Proof. (sketched) The proof of the existence result is based on introducing the following penalized systems

$$
\begin{gathered}
\partial_{t} u-D_{1} \Delta u-\operatorname{div}\left(\mathcal{A}_{11}^{h}\left(u^{+}, v^{+}\right) \nabla u+\mathcal{A}_{12}^{h}\left(u^{+}, v^{+}\right) \nabla v\right)=F^{h}\left(u^{+}, v^{+}\right), \\
\partial_{t} v-D_{2} \Delta v-\operatorname{div}\left(\mathcal{A}_{21}^{h}\left(u^{+}, v^{+}\right) \nabla u+\mathcal{A}_{22}^{h}\left(u^{+}, v^{+}\right) \nabla v\right)=G^{h}\left(u^{+}, v^{+}\right), \quad(x, t) \in Q_{T}:=\Omega \times(0, T], \\
\frac{\partial u}{\partial \eta}=\frac{\partial v}{\partial \eta}=0, u(x, 0)=u_{0}(x), \quad v(x, 0)=v_{0}(x) .
\end{gathered}
$$

Here for all $h>0$, one can choose e.g. $\mathcal{A}^{h}\left(r^{+}, s^{+}\right)=\mathcal{A}\left(\min \left\{r^{+}, \frac{1}{h}\right\}, \min \left\{s^{+}, \frac{1}{h}\right\}\right)$, and apply the same truncation procedure to $F, G$ in order to obtain $F^{h}, G^{h}$. In particular, $\mathcal{A}^{h}$ and $F^{h}, G^{h}$ are $L^{\infty}$ functions that approximate $\mathcal{A}$ and $F, G$, respectively, in $C^{2}(K)$, for all compact $K$ in $\mathbb{R}^{2}$. These approximate problems being uniformly parabolic, and existence of an $L^{2}\left(0, T ; H^{1}(\Omega)\right)$ solution is well known. Moreover, since $u_{0}, v_{0} \geq 0$, using the test functions $u^{-}, v^{-}$and assumptions $1.5,(1.8)$ one easily shows the non-negativity of $u$ and $v$ solving (2.1).

Let us denote the solution of (2.1) by $\mathbf{u}_{h}=\left(u_{h}, v_{h}\right)$ and sketch a version of the passage to the limit argument that we will reproduce at the discrete level. We have

$$
\left(u_{h}, v_{h}\right) \in\left(L^{2}\left(0, T ; H^{1}(\Omega)\right) \cap C\left(0, T ; L^{2}(\Omega)\right)\right)^{2} \text { with }\left\|\mathcal{A}^{h}\left(u_{h}, v_{h}\right)\right\|\left(\left|\nabla u_{h}\right|^{2}+\left|\nabla v_{h}\right|^{2}\right) \in L^{1}\left(Q_{T}\right)
$$

with a uniform estimate of the norms. Indeed, multiplication of the first and the second equation in 2.1. (where $u$ and $v$ are replaced by $u_{h}$ and $v_{h}$ respectively) by $u_{h}$ and $v_{h}$, respectively, gives

$$
\begin{aligned}
& \left\|u_{h}\right\|_{L^{\infty}\left(0, T ; L^{2}(\Omega)\right)}+\left\|v_{h}\right\|_{L^{\infty}\left(0, T ; L^{2}(\Omega)\right)} \leq C, \\
& \left\|\nabla u_{h}\right\|_{L^{2}\left(Q_{T}\right)}+\left\|\nabla v_{h}\right\|_{L^{2}\left(Q_{T}\right)} \leq C, \\
& \iint_{Q_{T}}\left(\max _{1 \leq i, j \leq 2}\left|\mathcal{A}_{i j}^{h}\left(u_{h}, v_{h}\right)\right|\right)\left(\left|\nabla u_{h}\right|^{2}+\left|\nabla v_{h}\right|^{2}\right) d x d t \leq C,
\end{aligned}
$$

where $C$ is independent of $h$. Here assumption (1.6) is used (notice that $\mathcal{A}^{h}$ inherits the structure properties of $\mathcal{A}$ ). Then we use the following version of the compactness lemma of [34].

Lemma 2.3 (Kruzhkov [34; see also [5]).

Let $\Omega$ be an open domain in $\mathbb{R}^{l}, T>0, Q_{T}=(0, T) \times \Omega$. Assume that the families of functions $\left(w^{h}\right)_{h},\left(F_{\alpha}^{h}\right)_{h, \alpha}$ are bounded in $L^{1}\left(Q_{T}\right)$ and satisfy $\frac{\partial}{\partial t} w^{h}=\sum_{|\alpha| \leq m} D^{\alpha} F_{\alpha}^{h}$ in $\mathcal{D}^{\prime}\left(Q_{T}\right)$. Assume that $w^{h}$ can be extended outside $Q_{T}$, and one has

$$
\sup _{|d x| \leq \Delta} \iint_{Q_{T}}\left|w^{h}(t, x+d x)-w^{h}(t, x)\right| d x d t \leq \omega(\Delta), \quad \text { with } \lim _{\Delta \rightarrow 0} \omega(\Delta)=0,
$$


where $\omega(\cdot)$ does not depend on $h$. Then $\left(w^{h}\right)_{h}$ is relatively compact in $L^{1}\left(Q_{T}\right)$.

Clearly, one can apply this lemma locally inside $[0, T] \times \Omega$.

From 2.2, it is easy to derive a uniform space translation estimate of the form 9.11) for $u_{h}$ and $v_{h}$. Further, using the sharp Sobolev embedding and the interpolation between $L^{2}\left(0, T ; L^{2^{*}}(\Omega)\right)$ and $L^{\infty}\left(0, T ; L^{2}(\Omega)\right)$, from the growth assumption (1.7) we infer that the quantities $\mathcal{A}_{i j}^{h}\left(u_{h}, v_{h}\right)$ are bounded in $L^{1}\left(Q_{T}\right)$ (moreover, they are equi-integrable in $\left.Q_{T}\right)$. Now uniform $L^{1}$ estimates on $u_{h}, v_{h}$, for the fluxes (in particular, the estimates for the cross-diffusion $\mathcal{A}^{h}\left(\mathbf{u}_{h}\right) \nabla \mathbf{u}_{h}$ ) and for the reaction terms are easily obtained from 2.2 and from the Cauchy-Schwarz inequality.

Therefore, using the weak form of equations (2.1), by Lemma 2.3 we infer that $u_{h}$ and $v_{h}$ converge (up to extraction of a subsequence) to some limits $u$ and $v$ a.e. in $Q_{T}$. Now by the Vitali theorem, from the aforementioned equi-integrability property we infer that $\mathcal{A}_{i j}^{h}\left(u_{h}, v_{h}\right)$ converges to $\mathcal{A}_{i j}(u, v)$ strongly in $L^{1}\left(Q_{T}\right)$.

In order to conclude the proof, we rewrite the cross-diffusion terms under the form

$$
\sqrt{\mathcal{A}_{11}}\left(\sqrt{\mathcal{A}_{11}} \nabla u\right), \quad \operatorname{sign} \mathcal{A}_{12} \sqrt{\left|\mathcal{A}_{12}\right|}\left(\sqrt{\left|\mathcal{A}_{12}\right|} \nabla v\right)
$$

and so on. Then we consider the terms

$$
\begin{array}{ll}
\sqrt{\mathcal{A}_{11}^{h}\left(u_{h}, v_{h}\right)} \nabla u_{h}, & \sqrt{\left|\mathcal{A}_{12}^{h}\left(u_{h}, v_{h}\right)\right|} \nabla v_{h}, \\
\sqrt{\left|\mathcal{A}_{21}^{h}\left(u_{h}, v_{h}\right)\right|} \nabla u_{h}, & \sqrt{\mathcal{A}_{22}^{h}\left(u_{h}, v_{h}\right)} \nabla v_{h}
\end{array}
$$

in the approximate weak formulation; thanks to $(2.2)$, these terms are all bounded in $L^{2}\left(Q_{T}\right)$. Their weak $L^{2}$ limits (along a subsequence) can be identified, via the weak $L^{1}\left(Q_{T}\right)$ convergence, with the terms

$$
\begin{array}{ll}
\sqrt{\mathcal{A}_{11}(u, v)} \nabla u, & \sqrt{\left|\mathcal{A}_{12}(u, v)\right|} \nabla v, \\
\sqrt{\left|\mathcal{A}_{21}(u, v)\right|} \nabla u, & \sqrt{\mathcal{A}_{22}(u, v)} \nabla v,
\end{array}
$$

respectively; here we use the strong $L^{2}\left(Q_{T}\right)$ compactness of $\left(\sqrt{\left|\mathcal{A}_{i j}^{h}\left(u_{h}, v_{h}\right)\right|}\right)_{h}$ and the weak $L^{2}\left(0, T ; H^{1}(\Omega)\right)$ compactness of $\left(u_{h}\right)_{h},\left(v_{h}\right)_{h}$. Using once more the strong $L^{2}$ compactness of $\left(\operatorname{sign} \mathcal{A}_{i j}^{h}\left(u_{h}, v_{h}\right) \sqrt{\left|\mathcal{A}_{i j}^{h}\left(u_{h}, v_{h}\right)\right|}\right)_{h}$, we are in a position to pass to the limit in the cross-diffusion terms of the weak formulation of (2.1). Finally, the a.e. convergence of $u_{h}, v_{h}$ to $u, v$ and the $L^{r}\left(Q_{T}\right), r>2$, bound on $u_{h}, v_{h}$ permit us to pass to the strong limit in the reaction terms $F^{h}\left(u_{h}, v_{h}\right)$ and $G^{h}\left(u_{h}, v_{h}\right)$. Thus we infer that $\mathbf{u}:=(u, v)$ is a nonnegative weak solution of $11.1,1.2$.

Remark 2.1. In the case of space dimension $l=3$, the growth assumption (1.7) can be relaxed in some particular cases. For instance, if the lower bound $\|\mathcal{A}(u, v)\| \geq \frac{1}{C}\left(u^{r}+v^{r}\right)$ holds with $r=10 / 3$, we can use estimates $\left(2.2\right.$ to get $L^{2}\left(Q_{T}\right)$ bounds of $\nabla u^{r / 2+1}, \nabla v^{r / 2+1}$. Using the optimal Sobolev embedding $H^{1}(\Omega) \subset L^{2}(\Omega)$, we infer a uniform $L^{2}\left(0, T ; L^{(r / 2+1) 2^{*}}(\Omega)\right)$ bound on $u_{h}$, $v_{h}$. Then the interpolation with $L^{\infty}\left(0, T ; L^{2}(\Omega)\right)$ allows to take a higher value of $r$ in $(1.7)$.

Bootstrapping this argument, eventually we can allow for $\|\mathcal{A}(u, v)\|$ with polynomial growth of order $r<4$ at infinity, as in the case of dimension $l=2$.

\section{Finite Volume Approximation}

We assume that $\Omega \subset \mathbb{R}^{l}, l=2$ (respectively, $l=3$ ) is an open bounded polygonal (resp., polyhedral) connected domain with boundary $\partial \Omega$. Following [20], we consider a family $\mathcal{T}_{h}$ of admissible meshes of the domain $\Omega$ consisting of disjoint open and convex polygons (resp., polyhedra) called control volumes. The parameter $h$ has the sense of an upper bound for the maximum diameter of the control volumes in $\mathcal{T}_{h}$. Whenever $\mathcal{T}_{h}$ is fixed, we will drop the subscript $h$ in the notation.

A generic volume in $\mathcal{T}$ is denoted by $K$. Because we consider the zero-flux boundary condition, we do not need to distinguish between interior and exterior control volumes; only inner interfaces between volumes are needed in order to formulate the scheme. 


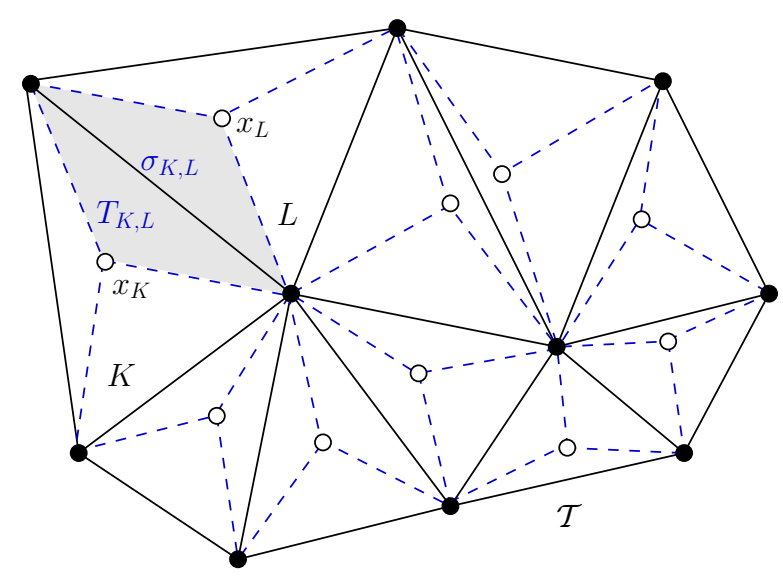

Figure 1. Control volumes, centers and diamonds (in dashed lines).

For all $K \in \mathcal{T}$, we denote by $|K|$ the $l$-dimensional Lebesgue measure of $K$. For all $K \in \mathcal{T}$, we denote by $N(K)$ the set of the neighbors of $K$ (i.e. the set of control volumes of $\mathcal{T}$ which have a common interface with $K$ ); a generic neighbor of $K$ is often denoted by $L$. For all $L \in N(K)$, we denote by $\sigma_{K, L}$ the interface between $K$ and $L$; we denote by $\eta_{K, L}$ the unit normal vector to $\sigma_{K, L}$ outward to $K$. We have $\eta_{L, K}=-\eta_{K, L}$. For an interface $\sigma_{K, L},\left|\sigma_{K, L}\right|$ will denote its $(l-1)$-dimensional measure.

By saying that $\mathcal{T}$ is admissible, we mean that there exists a family $\left(x_{K}\right)_{K \in \mathcal{T}}$ such that the straight line $\overline{x_{K} x_{L}}$ is orthogonal to the interface $\sigma_{K, L}$. The point $x_{K}$ is referred to as the center of $K$ (notice that in general, $x_{K}$ need not belong to $K$ ). In the case where $\mathcal{T}$ is a simplicial mesh of $\Omega$ (a triangulation, in dimension $l=2$ ), one takes for $x_{K}$ the center of the circumscribed ball of $K$. We also require that $\eta_{K, L} \cdot\left(x_{L}-x_{K}\right)>0$ (in the case of simplicial meshes, this restriction amounts to the Delaunay condition, see e.g. [20]). The "diamond" constructed from the neighbor centers $x_{K}, x_{L}$ and the interface $\sigma_{K, L}$ is denoted by $T_{K, L}$; e.g. in the case $x_{K} \in K, x_{L} \in L, T_{K, L}$ is the convex hull of $x_{K}, x_{L}$ and $\sigma_{K, L}$ ) (see Figure 11. We have $\Omega=\cup_{K \in \mathcal{T}}\left(\cup_{L \in N(K)} \bar{T}_{K, L}\right)$.

We denote by $d_{K, L}$ the distance between $x_{K}$ and $x_{L}$. We require local regularity restrictions on the family of meshes $\mathcal{T}_{h}$; namely,

$$
\begin{gathered}
\exists \gamma>0 \quad \forall h \quad \forall K \in \mathcal{T}_{h} \forall L \in N(K) \quad \operatorname{diam}(K)+\operatorname{diam}(L) \leq \gamma d_{K, L}, \\
\exists \gamma>0 \quad \forall h \quad \forall K \in \mathcal{T}_{h} \forall L \in N(K) \quad\left|\sigma_{K, L}\right| d_{K, L} \leq \gamma|K| .
\end{gathered}
$$

The first restriction is used in the proof of Lemma 9.2 which ensures discrete $L^{1}$ compactness. The second restriction is used in the proof of the discrete Sobolev embeddings (Proposition 9.3), and it can be bypassed (see Remark 5.1).

A discrete function on the mesh $\mathcal{T}_{h}$ is a set $\left(w_{K}\right)_{K \in \mathcal{T}_{h}}$. Whenever convenient, we identify it with the piecewise constant function $w_{h}$ on $\Omega$ such that $\left.w_{h}\right|_{K}=w_{K}$. Finally, the discrete gradient $\nabla_{h} w_{h}$ of a constant per control volume function $w_{h}$ is defined as the constant per diamond $T_{K, L}$ function, $\mathbb{R}^{l}$-valued, with the values

$$
\left.\left(\nabla_{h} w_{h}\right)\right|_{T_{K, L}}=\nabla_{K, L} w_{h}:=l \frac{w_{L}-w_{K}}{d_{K, L}} \eta_{K, L}
$$

3.1. Formulation of the scheme and the main result. To formulate the underlying scheme, we choose an admissible discretization of $Q_{T}$ consisting of an admissible mesh $\mathcal{T}_{h}$ of $\Omega$ and of a time step size $\Delta t_{h}>0$; both $\Delta t_{h}$ and the size $\max _{K \in \mathcal{T}_{h}} \operatorname{diam}(K)$ tend to zero as $h \rightarrow 0$. We define $N_{h}>0$ as the smallest integer such that $\left(N_{h}+1\right) \Delta t_{h} \geq T$, and set $t^{n}:=n \Delta t_{h}$ for $n \in\left\{0, \ldots, N_{h}\right\}$. Whenever $\Delta t_{h}$ is fixed, we will drop the subscript $h$ in the notation. 
The terms $F_{K}^{n+1}, G_{K}^{n+1}$ will approximate the averages

$$
\frac{1}{|K|} \int_{K} F\left(u\left(x, t^{n}\right), v\left(x, t^{n}\right)\right) d x, \quad \frac{1}{|K|} \int_{K} G\left(u\left(x, t^{n}\right), v\left(x, t^{n}\right)\right) d x,
$$

and are defined by

$$
F_{K}^{n+1}=F\left(u_{K}^{n+1^{+}}, v_{K}^{n+1^{+}}\right), \quad G_{K}^{n+1}=G\left(u_{K}^{n+1^{+}}, v_{K}^{n+1^{+}}\right) .
$$

In order to approximate the diffusive terms, we introduce the terms $\mathcal{A}_{i j, K}^{n+1}$. Herein, we make the choice

$$
\mathcal{A}_{i j, K, L}^{n+1}:=\mathcal{A}_{i j}\left(\min \left\{u_{K}^{n+1^{+}}, u_{L}^{n+1^{+}}\right\}, \min \left\{v_{K}^{n+1^{+}}, v_{L}^{n+1^{+}}\right\}\right)
$$

Remark 3.1. Let us stress that the choice of the minimum in the discretization of $\mathcal{A}_{12, K, L}^{n+1}, \mathcal{A}_{21, K, L}^{n+1}$ is imposed in order to justify the non-negativity property (see property (1.5) and the proof of Lemma 4.1 below). Then the choice of the diagonal terms $\mathcal{A}_{11, K, L}^{n+1}, \mathcal{A}_{22, K, L}^{n+1}$ is made in order to preserve, at the discrete level, the structure of the cross-diffusion matrix $\mathcal{A}$, namely the coercivity property (1.6). It should be pointed out that the above scheme actually approximates the penalized system $(2.1) ;$ the nonnegativity Lemma 4.1 is necessary in order to conclude that the scheme does approximate (1.1).

In practice, if one takes e.g. the simple scheme with

$$
\mathcal{A}_{12, K, L}^{n+1}:=\frac{u_{K}^{n+1}+u_{L}^{n+1}}{2}, \mathcal{A}_{21, K, L}^{n+1}:=\frac{v_{K}^{n+1}+v_{L}^{n+1}}{2}, \mathcal{A}_{i i, K, L}^{n+1}:=\frac{u_{K}^{n+1}+u_{L}^{n+1}+v_{K}^{n+1}+v_{L}^{n+1}}{2}, i=1,2,
$$

then no negative values appear in the numerical studies that we have made.

The computation starts from the initial cell averages

$$
u_{K}^{0}=\frac{1}{|K|} \int_{K} u_{0}(x) d x, \quad v_{K}^{0}=\frac{1}{|K|} \int_{K} v_{0}(x) d x .
$$

To advance the numerical solution from $t^{n}$ to $t^{n+1}=t^{n}+\Delta t$, we use the following implicit finite volume scheme: Determine $\left(u_{K}^{n+1}\right)_{K \in \mathcal{T}_{h}},\left(v_{K}^{n+1}\right)_{K \in \mathcal{T}_{h}}$ such that

$$
\begin{aligned}
|K| \frac{u_{K}^{n+1}-u_{K}^{n}}{\Delta t}-D_{1} & \sum_{L \in N(K)} \frac{\left|\sigma_{K, L}\right|}{d_{K, L}}\left(u_{L}^{n+1}-u_{K}^{n+1}\right) \\
& -\sum_{L \in N(K)} \frac{\left|\sigma_{K, L}\right|}{d_{K, L}}\left[\mathcal{A}_{11, K, L}^{n+1}\left(u_{L}^{n+1}-u_{K}^{n+1}\right)+\mathcal{A}_{12, K, L}^{n+1}\left(v_{L}^{n+1}-v_{K}^{n+1}\right)\right]=|K| F_{K}^{n+1} \\
|K| \frac{v_{K}^{n+1}-v_{K}^{n}}{\Delta t}-D_{2} & \sum_{L \in N(K)} \frac{\left|\sigma_{K, L}\right|}{d_{K, L}}\left(v_{L}^{n+1}-v_{K}^{n+1}\right) \\
& -\sum_{L \in N(K)} \frac{\left|\sigma_{K, L}\right|}{d_{K, L}}\left[\mathcal{A}_{21, K, L}^{n+1}\left(u_{L}^{n+1}-u_{K}^{n+1}\right)+\mathcal{A}_{22, K, L}^{n+1}\left(v_{L}^{n+1}-v_{K}^{n+1}\right)\right]=|K| G_{K}^{n+1}
\end{aligned}
$$

for all $K \in \mathcal{T}_{h}$ and $n \in\left[0, N_{h}\right]$. As usual, the homogeneous Neumann boundary condition is taken into account implicitly. Indeed, the parts of $\partial K$ that lie in $\partial \Omega$ do not contribute to the $\sum_{L \in N(K)}$ terms, which means that the flux zero is imposed on the external edges of the mesh.

The set of values $\left(u_{K}^{n+1}, v_{K}^{n+1}\right)_{K \in \mathcal{T}, n \in\left[0, N_{h}\right]}$ satisfying (3.6), (3.7) and (3.8) will be called a discrete solution. Whenever convenient, we will assimilate a discrete solution of the scheme at the time step $n$ to the couple $\mathbf{u}_{h}^{n+1}=\left(u_{h}^{n+1}, v_{h}^{n+1}\right)$ of piecewise constant on $\Omega$ functions given by

$$
\left.\forall K \in \mathcal{T}_{h} \forall n \in\left[0, N_{h}\right] \quad u_{h}^{n+1}\right|_{K}=u_{K}^{n+1},\left.\quad v_{h}^{n+1}\right|_{K}=v_{K}^{n+1} .
$$


We will write $\mathbf{u}_{h}$ for the discrete solution on $Q_{T}$, assimilated to the piecewise constant function

$$
\left(\sum_{K \in \mathcal{T}_{h}, n \in\left[0, N_{h}\right]} u_{K}^{n+1} \mathbb{1}_{\left(t^{n}, t^{n+1}\right] \times K}, \sum_{K \in \mathcal{T}_{h}, n \in\left[0, N_{h}\right]} v_{K}^{n+1} \mathbb{1}_{\left(t^{n}, t^{n+1}\right] \times K}\right) .
$$

Because in practical computations, one does not let the discretization parameter go to zero but fixes it to some small value, let us indicate the following mild restriction on the time step:

$$
\Delta t<\sup \left\{\frac{1}{2 a_{1}}, \frac{1}{2 a_{2}}\right\}
$$

which will be used to prove the existence of solutions to the scheme.

Our main result is

Theorem 3.1. Assume that $u_{0} \in\left(L^{2}(\Omega)\right)^{+}$and $v_{0} \in\left(L^{2}(\Omega)\right)^{+}$. Assume that the cross-diffusion matrix $\mathcal{A}$ fulfills (1.5), (1.6) and (1.7). Let $\mathbf{u}_{h}$ be the discrete solution generated by the finite volume scheme (3.6), (3.7) and (3.8) on a family of meshes satisfying (3.1), (3.2). Then, as $h \rightarrow 0$, $\mathbf{u}_{h}$ converges (along a subsequence) a.e. on $Q_{T}$ to a limit $\mathbf{u}=(u, v)$ that is a weak solution $\mathbf{u}$ of $1.1,1.2$.

\section{A PRIORI ESTimates AND EXISTEnCE}

4.1. Nonnegativity. We have the following lemma.

Lemma 4.1. Let $\left(u_{K}^{n+1}, v_{K}^{n+1}\right)_{K \in \mathcal{T}, n \in\left[0, N_{h}\right]}$ be a solution of the finite volume scheme (3.6), (3.7) and (3.8). Then, $\left(u_{K}^{n+1}, v_{K}^{n+1}\right)_{K \in \mathcal{T}, n \in\left[0, N_{h}\right]}$ is nonnegative.

Proof. Let us prove, by induction, that for all $n \in\left[0, N_{h}\right], \min \left\{u_{K}^{n+1}\right\}_{K \in \mathcal{T}_{h}} \geq 0$. The proof for the component $v$ is completely analogous.

Recall that $u_{K}^{0} \geq 0$ for all $K$. For $n \geq 0$, we fix $K$ such that $u_{K}^{n+1}=\min \left\{u_{L}^{n+1}\right\}_{L \in \mathcal{T}_{h}}$. Multiplying equation 3.7 by $-\Delta t u_{K}^{n+1^{-}}$, we deduce

$$
\begin{aligned}
-|K| u_{K}^{n+1-}\left(u_{K}^{n+1}-u_{K}^{n}\right)= & -D_{1} \Delta t \sum_{L \in N(K)} \frac{\left|\sigma_{K, L}\right|}{d_{K, L}}\left(u_{L}^{n+1}-u_{K}^{n+1}\right) u_{K}^{n+1}- \\
& -\Delta t \sum_{L \in N(K)} \frac{\left|\sigma_{K, L}\right|}{d_{K, L}}\left[\mathcal{A}_{11, K, L}^{n+1}\left(u_{L}^{n+1}-u_{K}^{n+1}\right)+\mathcal{A}_{12, K, L}^{n+1}\left(v_{L}^{n+1}-v_{K}^{n+1}\right)\right] u_{K}^{n+1-} \\
& -\Delta t|K| F_{K}^{n+1} u_{K}^{n+1^{-}} .
\end{aligned}
$$

By the choice of $K$ and the non-negativity of $\mathcal{A}_{11, K, L}^{n+1}$, we have

$$
\Delta t \sum_{L \in N(K)} \frac{\left|\sigma_{K, L}\right|}{d_{K, L}}\left(D_{1}+\mathcal{A}_{11, K, L}^{n+1}\right)\left(u_{L}^{n+1}-u_{K}^{n+1}\right) u_{K}^{n+1-} \geq 0 .
$$

In addition, due to assumption 1.5 and to the choice 3.5 of $\mathcal{A}_{12, K, L}^{n+1}$, we have

$$
\Delta t \sum_{L \in N(K)} \frac{\left|\sigma_{K, L}\right|}{d_{K, L}} \mathcal{A}_{12, K, L}^{n+1}\left(v_{L}^{n+1}-v_{K}^{n+1}\right) u_{K}^{n+1-}=0,
$$

Similarly, by the definition of $F_{K}^{n+1}$ we have

$$
F_{K}^{n+1} u_{K}^{n+1^{-}}=\left(a_{1}-b_{1} u_{K}^{n+1^{+}}-c_{1} v_{K}^{n+1^{+}}\right) u_{K}^{n+1^{+}} u_{K}^{n+1^{-}}=0 .
$$

Finally we use the identity $u_{K}^{n+1}=\left(u_{K}^{n+1}\right)^{+}-\left(u_{K}^{n+1}\right)^{-}$and the nonnegativity of $u_{K}^{n}$ to deduce from (4.1)- 4.2 that $\left(u_{K}^{n+1}\right)^{-}=0$. By induction in $n$, we infer that

$$
0 \leq u_{L}^{n+1} \text { for all } n \in\left[0, N_{h}\right] \text { and } L \in \mathcal{T}_{h} \text {. }
$$




\subsection{Discrete a priori estimates.}

Proposition 4.2. Let $\left(u_{K}^{n+1}, v_{K}^{n+1}\right)_{K \in \mathcal{T}, n \in\left[0, N_{h}\right]}$, be a solution of the finite volume scheme (3.6), (3.7) and (3.8). Then there exist a constant $C>0$, depending on $\Omega, T,\left\|u_{0}\right\|_{2},\left\|v_{0}\right\|_{2}, a_{1}, a_{2}, D_{1}, D_{2}$ such that

$$
\begin{gathered}
\max _{\left[0, N_{h}\right]} \sum_{K \in \mathcal{T}_{h}}|K|\left|u_{K}^{n+1}\right|^{2}+\max _{\left[0, N_{h}\right]} \sum_{K \in \mathcal{T}_{h}}|K|\left|v_{K}^{n+1}\right|^{2} \leq C, \\
b_{1} \sum_{n=0}^{N_{h}} \sum_{K \in \mathcal{T}_{h}}|K|\left|u_{K}^{n+1}\right|^{3}+c_{2} \sum_{n=0}^{N_{h}} \sum_{K \in \mathcal{T}_{h}}|K|\left|v_{K}^{n+1}\right|^{3} \leq C, \\
\sum_{n=0}^{N_{h}} \Delta t \sum_{K \in \mathcal{T}} \sum_{L \in N(K)} \frac{\left|\sigma_{K, L}\right|}{d_{K, L}}\left|u_{K}^{n+1}-u_{L}^{n+1}\right|^{2}+\sum_{n=0}^{N_{h}} \Delta t \sum_{K \in \mathcal{T}} \sum_{L \in N(K)} \frac{\left|\sigma_{K, L}\right|}{d_{K, L}}\left|v_{K}^{n+1}-v_{L}^{n+1}\right|^{2} \leq C,
\end{gathered}
$$

and

$$
\sum_{n=0}^{N_{h}} \Delta t \sum_{K \in \mathcal{T}} \sum_{L \in N(K)} \frac{\left|\sigma_{K, L}\right|}{d_{K, L}} \max _{1 \leq i, j \leq 2}\left|\mathcal{A}_{11, K, L}^{n+1}\right|\left(\left|u_{K}^{n+1}-u_{L}^{n+1}\right|^{2}+\left|v_{K}^{n+1}-v_{L}^{n+1}\right|^{2}\right) \leq C .
$$

Proof. The estimates actually hold under the assumption 1.6 on $\mathcal{A}$.

We multiply the first and the second equation in (3.7) by $\Delta t u_{K}^{n+1}$ and $\Delta t v_{K}^{n+1}$, respectively, and add together the outcomes. Summing the resulting equation over $K$ and $n$ yields

$$
S_{1}+S_{2}+S_{3}+S_{4}=0
$$

where

$$
\begin{aligned}
S_{1}= & \sum_{n=0}^{N_{h}} \sum_{K \in \mathcal{T}}|K|\left(\left(u_{K}^{n+1}-u_{K}^{n}\right) u_{K}^{n+1}+\left(v_{K}^{n+1}-v_{K}^{n}\right) v_{K}^{n+1}\right), \\
S_{2}= & -\sum_{n=0}^{N_{h}} \Delta t \sum_{K \in \mathcal{T}} \sum_{L \in N(K)} \frac{\left|\sigma_{K, L}\right|}{d_{K, L}}\left(D_{1}\left(u_{L}^{n+1}-u_{K}^{n+1}\right) u_{K}^{n+1}+D_{2}\left(v_{L}^{n+1}-v_{K}^{n+1}\right) v_{K}^{n+1}\right), \\
S_{3}=- & \sum_{n=0}^{N_{h}} \Delta t \sum_{K \in \mathcal{T}} \sum_{L \in N(K)} \frac{\left|\sigma_{K, L}\right|}{d_{K, L}}\left(\left[\mathcal{A}_{11, K, L}^{n+1}\left(u_{L}^{n+1}-u_{K}^{n+1}\right)+\mathcal{A}_{12, K, L}^{n+1}\left(v_{L}^{n+1}-v_{K}^{n+1}\right)\right] u_{K}^{n+1}\right. \\
& \left.\quad+\left[\mathcal{A}_{21, K, L}^{n+1}\left(u_{L}^{n+1}-u_{K}^{n+1}\right)+\mathcal{A}_{22, K, L}^{n+1}\left(v_{L}^{n+1}-v_{K}^{n+1}\right)\right] v_{K}^{n+1}\right), \\
S_{4}=- & \sum_{n=0}^{N_{h}} \Delta t \sum_{K \in \mathcal{T}}|K|\left(F_{K}^{n+1} u_{K}^{n+1}+G_{K}^{n+1} v_{K}^{n+1}\right) .
\end{aligned}
$$

From the inequality " $a(a-b) \geq \frac{1}{2}\left(a^{2}-b^{2}\right)$ ", we obtain

$$
\begin{aligned}
S_{1} & =\sum_{n=0}^{N_{h}} \sum_{K \in \mathcal{T}}|K|\left(\left(u_{K}^{n+1}-u_{K}^{n}\right) u_{K}^{n+1}+\left(v_{K}^{n+1}-v_{K}^{n}\right) v_{K}^{n+1}\right) \\
& \geq \frac{1}{2} \sum_{n=0}^{N_{h}} \sum_{K \in \mathcal{T}}|K|\left(\left|u_{K}^{n+1}\right|^{2}-\left|u_{K}^{n}\right|^{2}+\left|v_{K}^{n+1}\right|^{2}-\left|v_{K}^{n}\right|^{2}\right) \\
& =\frac{1}{2} \sum_{K \in \mathcal{T}}|K|\left(\left|u_{K}^{N_{h}+1}\right|^{2}-\left|u_{K}^{0}\right|^{2}+\left|v_{K}^{N_{h}+1}\right|^{2}-\left|v_{K}^{0}\right|^{2}\right) .
\end{aligned}
$$


Gathering by edges, we obtain

$$
S_{2}=\sum_{n=0}^{N_{h}} \Delta t \sum_{K \in \mathcal{T}} \sum_{L \in N(K)} \frac{\left|\sigma_{K, L}\right|}{d_{K, L}}\left(\frac{D_{1}}{2}\left|u_{K}^{n+1}-u_{L}^{n+1}\right|^{2}+\frac{D_{2}}{2}\left|v_{K}^{n+1}-v_{L}^{n+1}\right|^{2}\right) .
$$

Similarly, using the property 1.6 and the fact that $\mathcal{A}_{i j, L, K}^{n+1}=\mathcal{A}_{i j, K, L}^{n+1}$, we infer

$$
\begin{aligned}
S_{3}=- & \sum_{n=0}^{N_{h}} \Delta t \sum_{K \in \mathcal{T}} \sum_{L \in N(K)} \frac{\left|\sigma_{K, L}\right|}{d_{K, L}}\left(\left[\mathcal{A}_{11, K, L}^{n+1}\left(u_{L}^{n+1}-u_{K}^{n+1}\right)+\mathcal{A}_{12, K, L}^{n+1}\left(v_{L}^{n+1}-v_{K}^{n+1}\right)\right] u_{K}^{n+1}\right. \\
& \left.+\left[\mathcal{A}_{21, K, L}^{n+1}\left(u_{L}^{n+1}-u_{K}^{n+1}\right)+\mathcal{A}_{22, K, L}^{n+1}\left(v_{L}^{n+1}-v_{K}^{n+1}\right)\right] v_{K}^{n+1}\right) \\
\geq & \frac{1}{2 C} \sum_{n=0}^{N_{h}} \Delta t \sum_{K \in \mathcal{T}_{h}} \sum_{L \in N(K)} \frac{\left|\sigma_{K, L}\right|}{d_{K, L}} \max _{1 \leq i, j \leq 2}\left|\mathcal{A}_{i j, K, L}^{n+1}\right|\left[\left|u_{L}^{n+1}-u_{K}^{n+1}\right|^{2}+\left|v_{L}^{n+1}-v_{K}^{n+1}\right|^{2}\right] .
\end{aligned}
$$

Now we use the nonnegativity of $u_{K}^{n+1}$ and $v_{K}^{n+1}$ and the expressions (1.3) of $F$, $G$ to deduce

$$
S_{4} \geq-\sum_{n=0}^{N_{h}} \Delta t \sum_{K \in \mathcal{T}}|K|\left(a_{1}\left|u_{K}^{n+1}\right|^{2}+a_{2}\left|v_{K}^{n+1}\right|^{2}\right)+\sum_{n=0}^{N_{h}} \Delta t \sum_{K \in \mathcal{T}}|K|\left(b_{1}\left|u_{K}^{n+1}\right|^{3}+c_{1}\left|v_{K}^{n+1}\right|^{3}\right) .
$$

Collecting the previous inequalities we obtain

$$
\begin{aligned}
& \frac{1}{2} \sum_{K \in \mathcal{T}}|K|\left(\left|u_{K}^{N_{h}+1}\right|^{2}-\left|u_{K}^{0}\right|^{2}+\left|v_{K}^{N_{h}+1}\right|^{2}-\left|v_{K}^{0}\right|^{2}\right)+\sum_{n=0}^{N_{h}} \Delta t \sum_{K \in \mathcal{T}}|K|\left(b_{1}\left|u_{K}^{n+1}\right|^{3}+c_{1}\left|v_{K}^{n+1}\right|^{3}\right) \\
& \quad+\sum_{n=0}^{N_{h}} \Delta t \sum_{K \in \mathcal{T}} \sum_{L \in N(K)} \frac{\left|\sigma_{K, L}\right|}{d_{K, L}}\left(\frac{D_{1}}{2}\left|u_{K}^{n+1}-u_{L}^{n+1}\right|^{2}+\frac{D_{2}}{2}\left|v_{K}^{n+1}-v_{L}^{n+1}\right|^{2}\right) \\
& \quad+\frac{1}{2 C} \sum_{n=0}^{N_{h}} \Delta t \sum_{K \in \mathcal{T}_{h}} \sum_{L \in N(K)} \frac{\left|\sigma_{K, L}\right|}{d_{K, L}} \max _{1 \leq i, j \leq 2}\left|\mathcal{A}_{i j, K, L}^{n+1}\right|\left[\left|u_{L}^{n+1}-u_{K}^{n+1}\right|^{2}+\left|v_{L}^{n+1}-v_{K}^{n+1}\right|^{2}\right] \\
& \leq \sum_{n=0}^{N_{h}} \Delta t \sum_{K \in \mathcal{T}_{h}}|K|\left(a_{1}\left|u_{K}^{n+1}\right|^{2}+a_{2}\left|v_{K}^{n+1}\right|^{2}\right) .
\end{aligned}
$$

In view of the discrete Gronwall inequality, 4.3 follows from 4.7). Consequently, 4.7) entails the estimates (4.4)- 4.6). This concludes the proof of Proposition 4.2

4.3. Existence of a solution for the finite volume scheme. The existence of a solution for the finite volume scheme is given in the following proposition.

Proposition 4.3. Let $\mathcal{T}_{h}$ be an admissible discretization of $Q_{T}$ and assume that (3.9) holds. Then the discrete problem (3.6), (3.7) and (3.8) admits at least one solution $\left(u_{K}^{n+1}, v_{K}^{n+1}\right)_{(K, n) \in \mathcal{T}_{h} \times\left[0, N_{h}\right]}$.

For a proof, which is by induction in the time step, the elliptic counterpart of the coercivity estimates of the previous subsection is used. At this stage, we need an upper bound on the time step $\Delta t$ that permits to control the contribution of the terms $a_{1} u, a_{2} v$ on the right-hand side by the terms coming from the discrete time differencing of $u$ and $v$, respectively. Condition $(3.9)$ is sufficient to do this.

As soon as the estimates are achieved, a homotopy/topological degree argument allows to deduce existence starting from the existence of a discrete solution for a linear coercive system, see [20] for details.

Notice that in the place of the below argument, based upon the Brouwer fixed point theorem in the version of Ref. [36], the topological degree argument can be used, following Ref. [20]. At a price 
of more involved estimates, 3.9 can be relaxed, but an upper bound on the time step is necessary in order to ensure existence of solution for a pure Neumann boundary problem.

Proof. First we introduce the Hilbert space

$$
E_{h}=H_{h}(\Omega) \times H_{h}(\Omega),
$$

of couples $\mathbf{u}_{h}=\left(u_{h}, v_{h}\right)$ of discrete functions on $\Omega$ under the norm

$$
\left\|\mathbf{u}_{h}\right\|_{E_{h}}^{2}:=\left|u_{h}\right|_{H_{h}(\Omega)}^{2}+\left|v_{h}\right|_{H_{h}(\Omega)}^{2}+\left\|u_{h}\right\|_{L^{2}(\Omega)}^{2}+\left\|v_{h}\right\|_{L^{2}(\Omega)}^{2},
$$

where the "discrete $H_{0}^{1}$ seminorm" $|\cdot|_{H_{h}(\Omega)}^{2}$ is given by

$$
\left|w_{h}\right|_{H_{h}(\Omega)}^{2}:=\frac{1}{2} \sum_{K \in \mathcal{T}_{h}} \sum_{L \in N(K)}\left|T_{K, L}\right|\left|\frac{w_{L}-w_{K}}{d_{K, L}}\right|^{2},
$$

and the $L^{2}(\Omega)$ norm of $w_{h}$ is given by

$$
\left\|w_{h}\right\|_{L^{2}(\Omega)}^{2}:=\sum_{K \in \mathcal{T}_{h}}|K|\left|w_{K}\right|^{2} .
$$

Let $\Phi_{h}=\left(\varphi_{h}, \xi_{h}\right) \in E_{h}$ and define the discrete bilinear forms

$$
\begin{gathered}
T_{h}\left(\mathbf{u}_{h}, \Phi_{h}\right)=\sum_{K \in \mathcal{T}_{h}}|K|\left(u_{K} \varphi_{K}+v_{K} \xi_{K}\right), \\
a_{1, h}\left(\mathbf{u}_{h}^{n+1}, \Phi_{h}\right)=\frac{1}{2} \sum_{K \in \mathcal{T}_{h}} \sum_{L \in N(K)} \frac{\left|\sigma_{K, L}\right|}{d_{K, L}}\left(\left(u_{L}^{n+1}-u_{K}^{n+1}\right)\left(\varphi_{L}^{n+1}-\varphi_{K}^{n+1}\right)+\left(v_{L}^{n+1}-v_{K}^{n+1}\right)\left(\xi_{L}^{n+1}-\xi_{K}^{n+1}\right)\right) .
\end{gathered}
$$

Similarly, for given matrices $\mathcal{A}_{h}^{n+1}:=\left(\left(\mathcal{A}_{i j, K, L}^{n+1}\right)_{1 \leq i, j \leq 2}\right)_{K \in \mathcal{T}_{h}, L \in N(K)}$, define the bilinear form

$$
\begin{aligned}
a_{2, h}\left(\mathcal{A}_{h}^{n+1} ; \mathbf{u}_{h}^{n+1}, \Phi_{h}\right)= & \frac{1}{2} \sum_{K \in \mathcal{T}_{h}} \sum_{L \in N(K)} \frac{\left|\sigma_{K, L}\right|}{d_{K, L}}\left[\mathcal{A}_{11, K, L}^{n+1}\left(u_{L}^{n+1}-u_{K}^{n+1}\right)\left(\varphi_{L}^{n+1}-\varphi_{K}^{n+1}\right)\right. \\
& +\mathcal{A}_{12, K, L}^{n+1}\left(u_{L}^{n+1}-u_{K}^{n+1}\right)\left(\varphi_{L}^{n+1}-\varphi_{K}^{n+1}\right)+\mathcal{A}_{21, K, L}^{n+1}\left(v_{L}^{n+1}-v_{K}^{n+1}\right)\left(\xi_{L}^{n+1}-\xi_{K}^{n+1}\right) \\
& \left.+\mathcal{A}_{22, K, L}^{n+1}\left(v_{L}^{n+1}-v_{K}^{n+1}\right)\left(\xi_{L}^{n+1}-\xi_{K}^{n+1}\right)\right] .
\end{aligned}
$$

Multiplying (3.7) and (3.8) by $\varphi_{K}$ and $\xi_{K}$, respectively, summing in $K \in \mathcal{T}_{h}$, we get the equation $\frac{1}{\Delta t}\left(T_{h}\left(\mathbf{u}_{h}^{n+1}, \Phi_{h}\right)-T_{h}\left(\mathbf{u}_{h}^{n}, \Phi_{h}\right)\right)+a_{1, h}\left(\mathbf{u}_{h}^{n+1}, \Phi_{h}\right)+a_{2, h}\left(\mathcal{A}_{h}^{n+1}\left(\mathbf{u}_{h}^{n+1}\right) ; \mathbf{u}_{h}^{n+1}, \Phi_{h}\right)+T_{h}\left(R^{h}\left(\mathbf{u}_{h}^{n+1}\right), \Phi_{h}\right)=0 ;$ here the entries $\mathcal{A}_{i j, K, L}^{n+1}$ of $\mathcal{A}_{h}^{n+1}$ are defined from $\mathbf{u}_{h}^{n+1}$ with the help of formulas 3.5), furthermore, $R^{h}\left(\mathbf{u}_{h}^{n+1}\right):=\left(F_{h}^{n+1}, G_{h}^{n+1}\right)$ with the discrete functions $F_{h}^{n+1}, G_{h}^{n+1}$ defined from $\mathbf{u}_{h}^{n+1}$ by formulas (3.4). It is clear that, $\mathbf{u}_{h}^{n}$ being given, there exists a solution $\mathbf{u}_{h}^{n+1}$ of the above equation if and only if there exists a discrete solution of $(3.7),(3.8)$ at the time step $(n+1)$. Now we define, by duality, the mapping $\mathcal{P}$ from $E_{h}$ into itself:

$$
\begin{aligned}
\forall \Phi_{h} \in E_{h} \quad\left[\mathcal{P}\left(\mathbf{u}_{h}^{n+1}\right), \Phi_{h}\right]=\frac{1}{\Delta t} & \left(T_{h}\left(\mathbf{u}_{h}^{n+1}, \Phi_{h}\right)-T_{h}\left(\mathbf{u}_{h}^{n}, \Phi_{h}\right)\right) \\
& +a_{1, h}\left(\mathbf{u}_{h}^{n+1}, \Phi_{h}\right)+a_{2, h}\left(\mathcal{A}_{h}^{n+1}\left(\mathbf{u}_{h}^{n+1}\right) ; \mathbf{u}_{h}^{n+1}, \Phi_{h}\right)+T_{h}\left(R^{h}\left(\mathbf{u}_{h}^{n+1}\right), \Phi_{h}\right) .
\end{aligned}
$$

The continuity of the mapping $\mathcal{P}$ follows from the continuity of the nonlinearities $F, G,\left(\mathcal{A}_{i j}\right)_{1<i, j<2}$ and from the continuity of $a_{h, 1}(\cdot, \cdot), a_{h, 2}\left(\mathcal{A}_{h}^{n+1} ; \cdot, \cdot\right)$ and $T_{h}(\cdot, \cdot)$. Now we are looking for $\mathbf{u}_{h}^{n+1} \in E_{h}$ such that $\mathcal{P}\left(\mathbf{u}_{h}^{n+1}\right)=0$. According to [36], in order to prove the existence of $\mathbf{u}_{h}^{n+1}$ it remains to show that

$$
\left[\mathcal{P}\left(\mathbf{u}_{h}^{n+1}\right), \mathbf{u}_{h}^{n+1}\right]>0 \quad \text { whenever }\left\|\mathbf{u}_{h}^{n+1}\right\|_{E_{h}}=r>0
$$


for a sufficiently large $r$. We observe that

$$
\begin{aligned}
{\left[\mathcal{P}\left(\mathbf{u}_{h}^{n+1}\right), \mathbf{u}_{h}^{n+1}\right]=} & \frac{1}{\Delta t} \sum_{K \in \mathcal{T}_{h}}|K|\left|u_{K}^{n+1}\right|^{2}+\frac{1}{\Delta t} \sum_{K \in \mathcal{T}_{h}}|K|\left|v_{K}^{n+1}\right|^{2} \\
& +a_{1, h}\left(\mathbf{u}_{h}^{n+1}, \mathbf{u}_{h}^{n+1}\right)+a_{2, h}\left(\mathcal{A}_{h}^{n+1}\left(\mathbf{u}_{h}^{n+1}\right) ; \mathbf{u}_{h}^{n+1}, \mathbf{u}_{h}^{n+1}\right)+T_{h}\left(R^{h}\left(\mathbf{u}_{h}^{n+1}\right), \mathbf{u}_{h}^{n+1}\right) \\
& -\frac{1}{\Delta t} \sum_{K \in \mathcal{T}_{h}}|K| u_{K}^{n} u_{K}^{n+1}-\frac{1}{\Delta t} \sum_{K \in \mathcal{T}_{h}}|K| v_{K}^{n} v_{K}^{n+1} .
\end{aligned}
$$

Using the definition of $F_{K}^{n+1}$ and $G_{K}^{n+1}$, estimate (1.6) and Young's inequality we deduce from 4.9

$$
\begin{aligned}
& {\left[\mathcal{P}\left(\mathbf{u}_{h}^{n+1}\right), \mathbf{u}_{h}^{n+1}\right] } \\
& \geq \frac{1}{\Delta t} \sum_{K \in \mathcal{T}_{h}}|K|\left|u_{K}^{n+1}\right|^{2}+\frac{1}{\Delta t} \sum_{K \in \mathcal{T}_{h}}|K|\left|v_{K}^{n+1}\right|^{2}+D_{1}\left\|u_{h}^{n+1}\right\|_{H_{h}(\Omega)}^{2}+D_{2}\left\|v_{h}^{n+1}\right\|_{H_{h}(\Omega)}^{2} \\
&+\frac{1}{2 C} \sum_{n=0}^{N_{h}} \Delta t \sum_{K \in \mathcal{T}_{h}} \sum_{L \in N(K)} \frac{\left|\sigma_{K, L}\right|}{d_{K, L}} \max _{1 \leq i, j \leq 2}\left|\mathcal{A}_{i j, K, L}^{n+1}\right|\left[\left|u_{L}^{n+1}-u_{K}^{n+1}\right|^{2}+\left|v_{L}^{n+1}-v_{K}^{n+1}\right|^{2}\right] \\
&-\frac{1}{2 \Delta t} \sum_{K \in \mathcal{T}_{h}}|K|\left|u_{K}^{n+1}\right|^{2}-\frac{1}{2 \Delta t} \sum_{K \in \mathcal{T}_{h}}|K|\left|u_{K}^{n}\right|^{2}-\frac{1}{2 \Delta t} \sum_{K \in \mathcal{T}_{h}}|K|\left|v_{K}^{n+1}\right|^{2}-\frac{1}{2 \Delta t} \sum_{K \in \mathcal{T}_{h}}|K|\left|v_{K}^{n}\right|^{2} \\
&-a_{1} \sum_{K \in \mathcal{T}_{h}}|K|\left|u_{K}^{n+1}\right|^{2}-a_{2} \sum_{K \in \mathcal{T}_{h}}|K|\left|v_{K}^{n+1}\right|^{2} \\
& \geq D_{1}\left\|u_{h}^{n+1}\right\|_{H_{h}(\Omega)}^{2}+D_{2}\left\|v_{h}^{n+1}\right\|_{H_{h}(\Omega)}^{2} \\
&+\left(\frac{1}{2 \Delta t}-a_{1}\right) \sum_{K \in \mathcal{T}_{h}}|K|\left|u_{K}^{n+1}\right|^{2}+\left(\frac{1}{2 \Delta t}-a_{2}\right) \sum_{K \in \mathcal{T}_{h}}|K|\left|v_{K}^{n+1}\right|^{2}-\operatorname{const}\left(\Delta t, \mathbf{u}_{h}^{n}\right) \\
& \geq \operatorname{const}\left(D_{1}, D_{2}, a_{1}, a_{2}, \Delta t\right)\left(\left|u_{h}^{n+1}\right|_{H_{h}(\Omega)}^{2}+\left|v_{h}^{n+1}\right|_{H_{h}(\Omega)}^{2}+\left\|u_{h}\right\|_{L^{2}(\Omega)}^{2}+\left\|v_{h}\right\|_{L^{2}(\Omega)}^{2}\right)-\operatorname{const}\left(\Delta t, \mathbf{u}_{h}^{n}\right) .
\end{aligned}
$$

The constant const $\left(D_{1}, D_{2}, a_{1}, a_{2}, \Delta t\right)$ in the above expression is greater than zero, provided (3.9) holds. This implies that 4.8 holds for $r$ large enough (recall that $\left\|\mathbf{u}_{h}^{n+1}\right\|_{E_{h}}=r$ ). By induction in $n$, we deduce the existence of at least one solution to the scheme 3.7 .

\section{Compactness arguments}

In this section, with the help of a discrete compactness tool inspired by Lemma 2.3 we justify that the family $\mathbf{u}_{\mathbf{h}}$ of discrete solutions constructed in Proposition 4.3 is relatively compact in $L^{1}\left(Q_{T}\right)$.

Denote by $\mathcal{A}^{h}$ the $2 \times 2$ matrix on $Q_{T}$ with the entries $\mathcal{A}_{i j}^{h}$ given by

$$
\mathcal{A}_{i j}^{h}:=\frac{1}{2} \sum_{n=0}^{N_{h}} \sum_{K \in \mathcal{T}_{h}} \sum_{L \in N(K)} \mathcal{A}_{i j, K, L}^{n+1} \mathbb{1}_{\left(t^{n}, t^{n+1}\right] \times T_{K, L}} .
$$

We have the following convergence results along a subsequence:

Proposition 5.1. There exists a couple $\mathbf{u} \in\left(L^{r}\left(Q_{T}\right)\right)^{2} \cap L^{2}\left(0, T ; H^{1}(\Omega)\right)^{2}$ (recall that $r$ is defined in 1.7) and a subsequence of $\mathbf{u}_{h}=\left(u_{h}, v_{h}\right)$, not labelled, such that, as $h \rightarrow 0$,

(i) $\mathbf{u}_{h} \rightarrow \mathbf{u}$ strongly in $L^{1}\left(Q_{T}\right)$ and a.e. in $Q_{T}$,

(ii) $\nabla_{h} \mathbf{u}_{h} \longrightarrow \nabla \mathbf{u}$ weakly in $\left(L^{2}\left(Q_{T}\right)\right)^{2 l}$,

(iii) $\mathcal{A}^{h} \nabla_{h} \mathbf{u}_{h} \longrightarrow \mathcal{A}(\mathbf{u}) \nabla \mathbf{u}$ weakly in $\left(L^{1}\left(Q_{T}\right)\right)^{2 l}$,

(iv) $\left(F\left(\mathbf{u}_{h}\right), G\left(\mathbf{u}_{h}\right)\right) \longrightarrow(F(\mathbf{u}), G(\mathbf{u}))$ weakly in $\left(L^{1}\left(Q_{T}\right)\right)^{2}$. 
The above convergence properties are the ones needed to conclude the convergence proof; actually, strong $L^{p}\left(Q_{T}\right)$ convergences of $\mathbf{u}_{h}$ and of the associated reaction terms hold, for adequate values of $p$. On the contrary, the convergence of the intra-species diffusion fluxes $\nabla_{h} \mathbf{u}_{h}$ and of the crossdiffusion fluxes $\mathcal{A}^{h} \nabla_{h} \mathbf{u}_{h}$ cannot be upgraded to a strong convergence. One possibility to ensure strong convergence of discrete gradients is to replace the schemes used in this paper by schemes with more careful construction of the discrete gradients (essentially, one needs that the discrete gradients be consistent with affine functions). Another possibility is to perform an a posteriori reconstruction of the gradients, e.g. as in Ref. [21].

Proof. We first apply Lemma 9.2 (see Appendix A), using the estimates shown in Proposition 4.2 .

The discrete solution $\left(\mathbf{u}_{h}^{n+1}\right)_{n \in\left[0, N_{h}\right]}$ being given, we may consider that the evolution of the first component $\left(u_{h}^{n+1}\right)_{n \in\left[0, N_{h}\right]}$ of the solution is governed by the system of discrete equations

$$
\frac{u_{K}^{n+1}-u_{K}^{n}}{\Delta t}=\frac{1}{|K|} \sum_{L \in N(K)}\left|\sigma_{K, L}\right| \overrightarrow{\mathcal{F}}_{K, L}^{n+1} \cdot \eta_{K, L}+f_{K}^{n+1}
$$

obtained from (3.7); here we have assigned

$$
\begin{aligned}
f_{K}^{n+1}:= & F\left(u_{K}^{n+1}, v_{K}^{n+1}\right)=u_{K}^{n+1}\left(a_{1}-b_{1} u_{K}^{n+1}-c_{1} v_{K}^{n+1}\right), \\
\overrightarrow{\mathcal{F}}_{K, L}^{n+1}:= & D_{1} \frac{u_{L}^{n+1}-u_{K}^{n+1}}{d_{K, L}} \eta_{K, L}+\mathcal{A}_{11, K, L}^{n+1} \frac{u_{L}^{n+1}-u_{K}^{n+1}}{d_{K, L}} \eta_{K, L}+\mathcal{A}_{12, K, L}^{n+1} \frac{v_{L}^{n+1}-v_{K}^{n+1}}{d_{K, L}} \eta_{K, L} \\
& \equiv \frac{1}{l}\left[D_{1} \nabla_{K, L} u_{h}^{n+1}+\mathcal{A}_{11, K, L}^{n+1} \nabla_{K, L} u_{h}^{n+1}+\mathcal{A}_{12, K, L}^{n+1} \nabla_{K, L} u_{h}^{n+1}\right] .
\end{aligned}
$$

Equations (5.1) have the form (9.8) required in Lemma 9.2.

It remains to check that the local $L^{1}$ bounds $(9.9),(9.10)$ are verified. We actually have the global $L^{1}\left(Q_{T}\right)$ uniform estimates on the families

$$
\begin{aligned}
u^{h}:=\sum_{n=0}^{N_{h}} u_{h}^{n+1} \mathbb{1}_{\left(t^{n}, t^{n+1}\right]}, & \overrightarrow{\mathcal{F}}_{h}:=\frac{1}{2} \sum_{n=0}^{N_{h}} \sum_{K \in \mathcal{T}_{h}} \sum_{L \in N(K)} \overrightarrow{\mathcal{F}}_{K, L}^{n+1} \mathbb{1}_{\left(t^{n}, t^{n+1}\right] \times T_{K, L}} \\
f^{h}:=\sum_{n=0}^{N_{h}} f_{h}^{n+1} \mathbb{1}_{\left(t^{n}, t^{n+1}\right]}, & \nabla_{h} u_{h}:=\frac{1}{2} \sum_{n=0}^{N_{h}} \sum_{K \in \mathcal{T}_{h}} \sum_{L \in N(K)}^{\nabla_{K, L} u_{h}^{n+1} \mathbb{1}_{\left(t^{n}, t^{n+1}\right] \times T_{K, L} .}} .
\end{aligned}
$$

Indeed, the quadratic growth of $F, G$ and the $L^{2}\left(Q_{T}\right)$ estimate on the discrete solutions, trivially derived from 4.3 , ensures the $L^{1}\left(Q_{T}\right)$ bound on $\left(f^{h}\right)_{h}$. Combined with the Cauchy-Schwarz inequality, the same bound yields the $L^{1}\left(Q_{T}\right)$ estimate of $\left(u^{h}\right)_{h}$.

As in the proof of Theorem 2.2, we now use the growth assumption (1.7) (here we can take advantage of the choice of the min in formulas (3.5) for $\mathcal{A}_{i j, K, L}^{n+1}$ ). Using the critical discrete Sobolev embedding (see Proposition 9.3 in Appendix B) and the interpolation between $L^{p_{t}}\left(0, T ; L^{p_{x}}(\Omega)\right.$ ) spaces, from the $L^{\infty}\left(0, T ; L^{2}(\Omega)\right)$ estimate (4.3) and the discrete $L^{2}\left(0, T ; H^{1}(\Omega)\right)$ estimate 4.5 we get a uniform $L^{r}\left(Q_{T}\right)$ bound on $u^{h}$ and a uniform $L^{1}\left(Q_{T}\right)$ bound on the terms $\mathcal{A}_{i j}^{h}$.

Finally, the estimate 4.6 is exactly the $L^{2}\left(Q_{T}\right)$ estimate of the product $\sqrt{\left|\mathcal{A}^{h}\right|} \nabla_{h} \mathbf{u}_{h}$ (in this abusive notation, we mean that the square root of $\mathcal{A}^{h}$ is taken componentwise). The two latter bounds permit to control $\mathcal{A}^{h} \mathbf{u}_{h}$ in $L^{1}\left(Q_{T}\right)$.

Now, because $\overrightarrow{\mathcal{F}}^{h}$ is precisely the first component of the vector $\frac{1}{l}\left[D_{1} \nabla_{h} \mathbf{u}_{h}+\mathcal{A}^{h} \nabla_{h} \mathbf{u}_{h}\right]$, and $D_{1} \nabla_{h} \mathbf{u}_{h}$ is $L^{2}\left(Q_{T}\right)$ bounded by estimate 4.5 , by the Cauchy-Schwarz inequality we infer a uniform $L^{1}\left(Q_{T}\right)$ estimate of $\overrightarrow{\mathcal{F}}^{h}$ and also the one of $\nabla_{h} u^{h}$. Thus $(9.9),(9.10)$ are verified; the uniform $L^{1}(\Omega)$ bound on the initial data $u_{h}^{0}$ is also clear from $(3.6)$, and Lemma 9.2 can be applied to derive the $L^{1}\left(Q_{T}\right)$ compactness of $\left(u^{h}\right)_{h}$.

The arguments for the second component of $\mathbf{u}_{h}$, denoted by $v^{h}$, is entirely similar. Thus we can define the limit $\mathbf{u}=(u, v)$ of (a subsequence of) $\mathbf{u}_{h}$ and obtain the claim (i).

Further, the claim (ii) is deduced from the estimate (4.5). Indeed, one uses (4.5) to bound $\nabla_{h} \mathbf{u}_{h}$ in $L^{2}\left(Q_{T}\right)$. Upon extraction of a further subsequence, we have e.g. $u^{h} \rightarrow u$ in $L^{2}\left(Q_{T}\right)$ and $\nabla_{h} u^{h} \rightarrow \chi$ 
in $\left(L^{2}\left(Q_{T}\right)^{l}\right.$, where $\chi$ has yet to be identified. For this purpose, one takes a smooth compactly supported vector-function $\phi$ on $Q_{T}$ and proves, using the definition $(3.3)$ together with the discrete summation-by-parts and the consistency of the finite volume approximation of $\operatorname{div} \phi$, that

$$
\int_{0}^{T} \int_{\Omega} \nabla u \cdot \phi=-\int_{0}^{T} \int_{\Omega} u \operatorname{div} \phi .
$$

This shows that $u \in L^{2}\left(0, T ; H^{1}(\Omega)\right)$ and that $\chi$ identifies with $\nabla u$ (cf. Ref. 24]; our discrete gradient is less elaborate than the one of Ref. 24, but the idea of the proof is the same). The proof for $\nabla_{h} v^{h}$ is identical.

Finally, the claims (iii),(iv) follow because the uniform $L^{2}\left(Q_{T}\right)$ estimates of $\mathbf{u}_{h}$ and of $\sqrt{\left|\mathcal{A}_{i j}^{h}\right|}$ (see the arguments above) can be upgraded to adequate $L^{p}\left(Q_{T}\right)$ estimates with $p>2$. Using in addition the quadratic growth of $F$ and the a.e. convergence of $\mathbf{u}_{h}$ to $\mathbf{u}$, by the Vitali theorem we get (iv). Similarly, we get the strong $L^{2}\left(Q_{T}\right)$ convergence of $\sqrt{\left|\mathcal{A}_{i j}^{h}\right|}$ to $\sqrt{\left|\mathcal{A}_{i j}(\mathbf{u})\right|}$. Then, as in the proof of Theorem 2.2 we pass to the limit first in $\sqrt{\left|\mathcal{A}_{i j}^{h}\right|} \nabla_{h} \mathbf{u}_{h}$ and then in $\mathcal{A}^{h} \nabla_{h} \mathbf{u}_{h}$; hence we get (iii).

Remark 5.1. In the above compactness proof, one can bypass the use of the discrete Sobolev embeddings of Appendix B, if one uses the $L^{3}\left(Q_{T}\right)$ estimate (4.4) (which is valid under the assumption $b_{1}, c_{2}>0$ ), and if the restriction $r<3$ is taken in (1.7). In this way, restriction (3.2) on the family of meshes can de dropped.

\section{Convergence Analysis}

Our final goal is to prove that the limit function $\mathbf{u}=(u, v)$ constructed in Proposition 5.1 constitute a weak solution of system (1.1). Let us pass to the limit in (3.7) to get the first equality in Definition 2.1 the arguments for the passage to the limit in (3.8) are entirely similar.

Let $\varphi \in \mathcal{D}([0, T) \times \bar{\Omega})$. Set $\varphi_{K}^{n}:=\varphi\left(t^{n}, x_{K}\right)$ for all $K \in \mathcal{T}_{h}$ and $n \in\left[0, N_{h}+1\right]$. Multiply the discrete equation (3.7) by $\Delta t \varphi_{K}^{n+1}$ and sum up in $K \in \mathcal{T}_{h}$ and $n \in\left[0, N_{h}\right]$. This yields

$$
S_{1}^{h}+S_{2}^{h}+S_{3}^{h}=S_{4}^{h},
$$

where

$$
\begin{aligned}
S_{1}^{h} & =\sum_{n=0}^{N_{h}} \sum_{K \in \mathcal{T}_{h}}|K|\left(u_{K}^{n+1}-u_{K}^{n}\right) \varphi_{K}^{n+1}, \\
S_{2}^{h} & =-\sum_{n=0}^{N_{h}} \Delta t \sum_{K \in \mathcal{T}_{h}} \sum_{L \in N(K)} \frac{\left|\sigma_{K, L}\right|}{d_{K, L}} D_{1}\left(u_{L}^{n+1}-u_{K}^{n+1}\right) \varphi_{K}^{n+1}, \\
S_{3}^{h} & =-\sum_{n=0}^{N_{h}} \Delta t \sum_{K \in \mathcal{T}} \sum_{L \in N(K)} \frac{\left|\sigma_{K, L}\right|}{d_{K, L}}\left[\mathcal{A}_{11, K, L}^{n+1}\left(u_{L}^{n+1}-u_{K}^{n+1}\right)+\mathcal{A}_{12, K, L}^{n+1}\left(v_{L}^{n+1}-v_{K}^{n+1}\right)\right] \varphi_{K}^{n+1}, \\
S_{4}^{h} & =\sum_{n=0}^{N_{h}} \Delta t \sum_{K \in \mathcal{T}}|K| F_{K}^{n+1} \varphi_{K}^{n+1} .
\end{aligned}
$$

Making summation by parts in time and keeping in mind that $\varphi_{K}^{N_{h}+1}=0$ for all $K \in \mathcal{T}_{h}$, by classical arguments (cf. [20]) we get

$$
\begin{aligned}
S_{1}^{h} & =-\sum_{n=0}^{N_{h}} \sum_{K \in \mathcal{T}_{h}}|K| u_{K}^{n+1}\left(\varphi_{K}^{n+1}-\varphi_{K}^{n}\right)-\sum_{K \in \mathcal{T}_{h}}|K| u_{K}^{0} \varphi_{K}^{0} \\
& =-\sum_{n=0}^{N_{h}} \sum_{K \in \mathcal{T}_{h}} \int_{t^{n}}^{t^{n+1}} \int_{K} u_{K}^{n+1} \partial_{t} \varphi\left(t, x_{K}\right) d x d t-\sum_{K \in \mathcal{T}_{h}} \int_{K} u_{0}(x) \varphi\left(0, x_{K}\right) d x=:-S_{1,1}^{h}-S_{1,2}^{h} .
\end{aligned}
$$


Next, we introduce

$$
S_{1}^{h, *}=-\sum_{n=0}^{N_{h}} \sum_{K \in \mathcal{T}_{h}} \int_{t^{n}}^{t^{n+1}} \int_{K} u^{h}(t, x) \partial_{t} \varphi(t, x) d x d t-\int_{\Omega} u_{0}(x) \varphi(0, x) d x=:-S_{1,1}^{h, *}-S_{1,2}^{h, *} .
$$

Then

$$
\left|S_{1,2}^{h}-S_{1,2}^{h, *}\right| \leq\left(\int_{\Omega}\left|u_{0}(x)\right|^{2} d x \sum_{K \in \mathcal{T}_{h}} \int_{K}\left|\varphi\left(0, x_{K}\right)-\varphi(0, x)\right|^{2} d x\right)^{1 / 2} \leq C h,
$$

due to the Lipschitz continuity of $\varphi$. Next, from the definition of the piecewise constant function $u^{h}$,

$$
\begin{aligned}
S_{1,1}^{h}-S_{1,1}^{h, *} & =\sum_{n=0}^{N_{h}} \sum_{K \in \mathcal{T}_{h}}\left(\int_{t^{n}}^{t^{n+1}} \int_{K} u_{K}^{n+1} \partial_{t} \varphi\left(t, x_{K}\right) d x d t-\int_{t^{n}}^{t^{n+1}} \int_{K} u_{K}^{n+1} \partial_{t} \varphi(t, x) d x d t\right) \\
& =\sum_{n=0}^{N_{h}} \sum_{K \in \mathcal{T}_{h}} u_{K}^{n+1} \int_{t^{n}}^{t^{n+1}} \int_{K}\left(\partial_{t} \varphi\left(t, x_{K}\right)-\partial_{t} \varphi(t, x)\right) d x d t
\end{aligned}
$$

Using again the regularity of $\partial_{t} \varphi$ and the Cauchy-Schwarz inequality, we obtain

$$
\left|S_{1,1}^{h}-S_{1,1}^{h, *}\right| \leq C h\left(\sum_{n=0}^{N_{h}} \Delta t \sum_{K \in \mathcal{T}_{h}}|K|\left|u_{K}^{n+1}\right|^{2}\right)^{1 / 2} \leq C h
$$

in view of the $L^{\infty}\left(0, T ; L^{2}(\Omega)\right)$ estimate 4.3$)$. Thus

$$
\lim _{h \rightarrow 0}\left|S_{1,2}^{h}-S_{1,2}^{h, *}\right|=0, \quad \lim _{h \rightarrow 0}\left|S_{1,1}^{h}-S_{1,1}^{h, *}\right|=0 .
$$

Then it is clear from Proposition 5.1(i) that, along a subsequence,

$$
\begin{gathered}
\lim _{h \rightarrow 0} S_{1}^{h}=\lim _{h \rightarrow 0} S_{1}^{h, *}=-\int_{0}^{T} \int_{\Omega} u \partial_{t} \varphi-\int_{\Omega} u_{0} \varphi(0, \cdot) . \\
\lim _{h \rightarrow 0} S_{1}^{h}=-\int_{0}^{T} \int_{\Omega} u \partial_{t} \varphi-\int_{\Omega} u_{0} \varphi(0, \cdot),
\end{gathered}
$$

along a subsequence. Indeed, the adaptation of the convergence argument of [20] starting from the $L^{1}$ compactness of $\left(u_{h}\right)_{h}$, instead of the $L^{2}$ compactness, is straightforward.

Further, again along a subsequence, we have

$$
\lim _{h \rightarrow 0} S_{2}^{h}=D_{1} \iint_{Q_{T}} \nabla u \cdot \nabla \varphi, \quad \lim _{h \rightarrow 0} S_{3}^{h}=\iint_{Q_{T}}\left(\mathcal{A}_{11}(u, v) \nabla u+\mathcal{A}_{12}(u, v) \nabla v\right) \cdot \nabla \varphi .
$$

Our proof is slightly different from the classical one (cf. [20]), adapted to the definition (3.3) of the discrete gradient and to the associated weak convergence statements of Proposition 5.1 items (ii) and (iii). Let us put forward the arguments for the term $S_{2}^{h}$. Gathering by edges and using the definition (3.3) of $\nabla_{h}$, we have

$$
\begin{aligned}
S_{2}^{h} & =\frac{D_{1}}{2} \sum_{n=0}^{N_{h}} \Delta t \sum_{K \in \mathcal{T}_{h}} \sum_{L \in N(K)} \frac{1}{l}\left|\sigma_{K, L}\right| d_{K, L} l \frac{u_{L}^{n+1}-u_{K}^{n+1}}{d_{K, L}} \frac{\varphi_{L}^{n+1}-\varphi_{K}^{n+1}}{d_{K, L}} \\
& =\frac{D_{1}}{2} \sum_{n=0}^{N_{h}} \Delta t \sum_{K \in \mathcal{T}_{h}} \sum_{L \in N(K)}\left|T_{K, L}\right|\left(\nabla_{K, L} u_{h}^{n+1} \cdot \eta_{K, L}\right)\left(\nabla \varphi\left(t^{n+1}, \overline{x_{K, L}}\right) \cdot \eta_{K, L}\right),
\end{aligned}
$$

where $\overline{x_{K, L}}$ is some point on the segment with the endpoints $x_{K}, x_{L}$. Moreover, because the values of $\nabla_{K, L}$ are directed by $\eta_{K, L}$, we actually have

$$
\left(\nabla_{K, L} u_{h}^{n+1} \cdot \eta_{K, L}\right)\left(\nabla \varphi\left(t^{n+1}, \overline{x_{K, L}}\right) \cdot \eta_{K, L}\right) \equiv \nabla_{K, L} u_{h}^{n+1} \cdot \nabla \varphi\left(t^{n+1}, \overline{x_{K, L}}\right)
$$



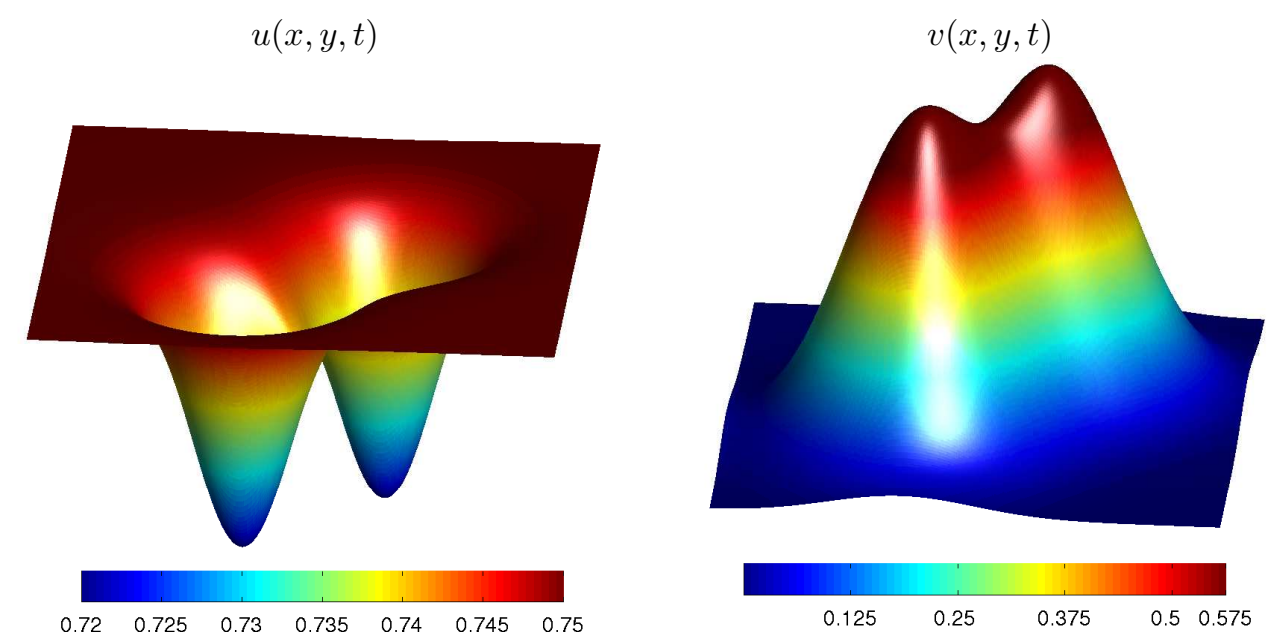

Figure 2. Lotka-Volterra model: Spread of a population at time $t=20$ for species $u, v$ (Example 1).

Since each term corresponding to $T_{K, L}$ appears twice in the above formula,

$$
S_{2}^{h}=D_{1} \int_{0}^{T} \int_{\Omega} \nabla_{h} u^{h} \cdot(\nabla \varphi)_{h}
$$

where

$$
\left.(\nabla \varphi)_{h}\right|_{\left(t^{n}, t^{n+1}\right] \times T_{K, L}}:=\nabla \varphi\left(t^{n+1}, \overline{x_{K, L}}\right) .
$$

Observe that from the continuity of $\nabla \varphi$ we get $(\nabla \varphi)_{h} \rightarrow \nabla \varphi$ in $L^{\infty}\left(Q_{T}\right)$. Hence using the weak $L^{1}$ convergence of $\nabla_{h} u^{h}$ to $\nabla u$, we pass to the limit in $S_{2}^{h}$, as $h \rightarrow 0$. The proof of the convergence claim for $S_{3}^{h}$ is entirely similar, using Proposition 5.1(iii) in the place of Proposition 5.1(ii).

Finally, using Proposition 5.1 (iv), we readily deduce that $S_{4}^{h}$ converges to $\iint_{Q_{T}} F(u, v) \varphi$ as $h \rightarrow 0$. Gathering the obtained results, we justify the first equality in Definition 2.1 and end the proof of Theorem 3.1 .

\section{Numerical ExAmples}

Numerical results presented hereafter refer to systems (1.1)-(1.2) and (1.4). In all cases the computations correspond to simple square domains, and numerical simulations are carried out using uniform meshes of $\mathcal{N}=65536$ control volumes.

Due to the lack of exact solutions for the forthcoming examples, we compute errors in different norms using a numerical solution on an extremely fine mesh as reference. To measure errors between such a reference solution $z_{\text {ref }}$ and an approximate solution $z_{h}$, at time $t^{n}$, we will use normalized $L^{p}$-errors:

where

$$
e_{p}^{n}=\frac{\left\|z_{\mathrm{ref}}^{n}-z_{h}^{n}\right\|_{p}}{\left\|z_{\mathrm{ref}}^{n}\right\|_{p}} \quad p=1,2, \infty
$$

$$
\left\|z_{\mathrm{ref}}^{n}-z_{h}^{n}\right\|_{\infty}=\max _{K \in \mathcal{T}_{h}}\left|z_{\mathrm{ref}, K}^{n}-z_{h K}^{n}\right| ; \quad\left\|z_{\mathrm{ref}}^{n}-z_{h}^{n}\right\|_{p}=\left(\frac{1}{|K|} \sum_{K \in \mathcal{T}_{h}}\left|z_{\mathrm{ref}, K}^{n}-z_{h K}^{n}\right|^{p}\right)^{1 / p}, \quad p=1,2 .
$$


Here $z_{\text {ref, } K}^{n}$ stands for the projection of the reference solution onto the control volume $K$. For the computation of convergence rates for the scalar field $z$, we herein use

$$
r(z)=\frac{\log \left(e(z) / e^{*}(z)\right)}{\log \left(h / h^{*}\right)},
$$

where $e(z)$ and $e^{*}(z)$ denote the respective errors computed for two consecutive meshes of sizes $h$ and $h^{*}$. For solving the corresponding nonlinear system arising from the implicit finite volume scheme, we have used the Newton method, where at each time step, only a few iterations are required to achieve convergence. In addition, the linear systems involved in Newton's method are solved by the GMRES method.

7.1. Example 1: Squirrel war without cross-diffusion. The first numerical test corresponds to the Lotka-Volterra system with diffusion (1.4) endowed with zero-flux boundary conditions. The spatial domain is $\bar{\Omega}=[-1,1]^{2}$ and to perform the numerical simulations we adopt a set of parameters used in [44]: $D_{1}=1 \mathrm{Km}^{2} /$ year, $D_{2}=18 \mathrm{Km}^{2} /$ year, $a_{1}=0.61 /$ year, $a_{2}=0.82 /$ year, $b_{1}=0.4575, b_{2}=$ $0.31, c_{1}=9.5, c_{2}=8.2$. The carrying capacities for both species are $a_{1} / b_{1}=0.75 /$ ha and $a_{2} / b_{2}=$ $10 /$ ha respectively. The initial distribution corresponds to the $u$-species at their constant carrying capacity $u_{0}(x)=0.75 /$ ha, and the $v$-species concentrated in small pockets at two spatial points. The system is evolved and a snapshot of the corresponding numerical solution at time $t=20$ is shown in Figure 2, It is observed that grey squirrels had spread outward and red squirrels started to recede. A numerical rate of convergence slightly under $h^{2}$ for different norms, is noticed from Table 1 and Figure 3(a).

\begin{tabular}{cccccccc}
\hline $\mathcal{N}$ & $h$ & $L^{1}$-error & $r^{1}(u)$ & $L^{2}$-error & $r^{2}(u)$ & $L^{\infty}$-error & $r^{\infty}(u)$ \\
\hline 1024 & $3.90 \times 10^{-3}$ & $1.35 \times 10^{-3}$ & - & $2.72 \times 10^{-3}$ & - & $1.21 \times 10^{-2}$ & - \\
4096 & $9.77 \times 10^{-4}$ & $1.01 \times 10^{-4}$ & 1.9021 & $2.01 \times 10^{-4}$ & 1.9131 & $9.12 \times 10^{-4}$ & 1.8917 \\
16384 & $2.44 \times 10^{-4}$ & $7.59 \times 10^{-6}$ & 1.8932 & $1.49 \times 10^{-5}$ & 1.9049 & $6.67 \times 10^{-5}$ & 1.9239 \\
65536 & $6.10 \times 10^{-5}$ & $5.65 \times 10^{-7}$ & 1.9046 & $1.11 \times 10^{-6}$ & 1.9108 & $4.92 \times 10^{-6}$ & 1.9126 \\
\hline
\end{tabular}

TABLE 1. Number of control volumes $\mathcal{N}$, meshsize $h$, approximate errors in different norms for $u$ and observed convergence rates $r$ at simulated time $t=20$ (Example 1 ).

7.2. Example 2: Squirrel war with cross-diffusion. As a second numerical example, consider model 1.1, 1.2, where we have chosen the same coefficients of intra- and inter-specific competition as in Example 1. From Figure 4, it can be seen that the system behaves in a qualitatively similar fashion as in Example 1. Notice however, from the snapshot of the $u$-species, that the resident species start to compete with the invading species, specifically near the gradient of density. A comparison of the species' behavior in both Examples 1 and 2 can be analyzed from Figure 5, where we display profiles of the numerical solutions at time $t=20$ in a $1 \mathrm{D}$ slice of the domain, namely the level $y=0$. The asynchrony of both species is clear from these plots. The concentration of resident species (in the left plot) in two small regions driven by cross diffusion suggest the creation of spatial niches that allows the survival of residents that would otherwise be completely depleted.

The corresponding convergence history for Example 2 is given in Table 2 and Figure $3(\mathrm{~b})$. It is clearly seen that the method provides an experimental rate of convergence between $h^{3 / 2}$ and $h^{2}$.

7.3. Example 3: Cross-diffusion and pattern formation. For the last example, all parameters are set as in Example 2, except for $a_{2}=0.52$ /year and the initial distribution for the species, which is now chosen to be a normally distributed random perturbation around the equilibrium state $\left(u^{*}, v^{*}\right)=$ $(0.0769,0.0605)$ on the entire domain, with a variance lower than the amplitude of the final patterns. The approach of perturbing an equilibrium point and studying the formation of nonuniform spatial patterns is by now standard (see e.g. [35]). The choice for the parameters is such that the sufficient 
(a)

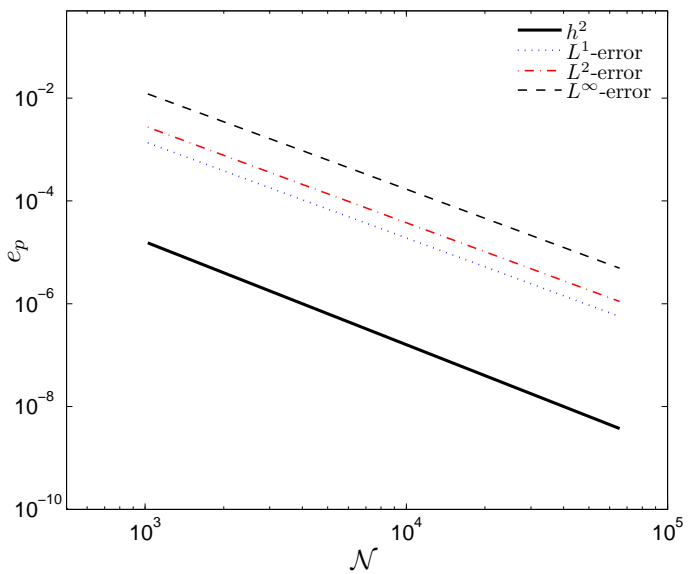

(b)

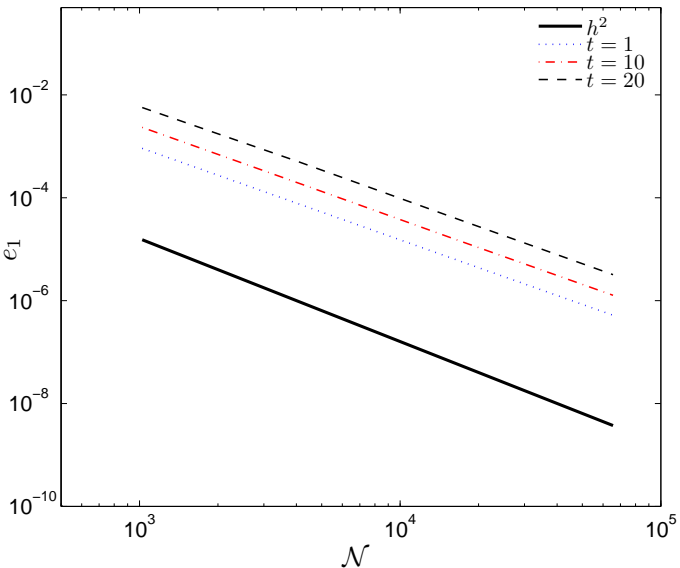

Figure 3. Meshsize $h$ and errors for $u$ in: (a) different norms versus the number of control volumes $\mathcal{N}$, where the simulated time is $t=20$ (Example 1); and (b) $L^{1}$-norm versus the number of control volumes $\mathcal{N}$ for different simulated times (Example 2).
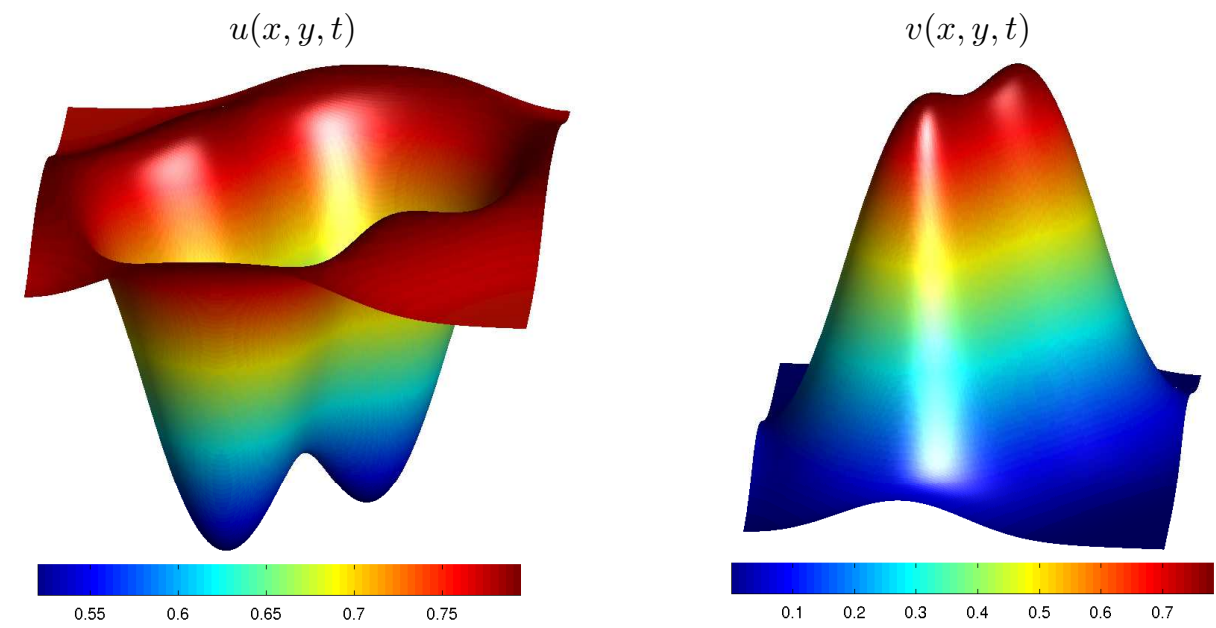

Figure 4. Model with cross-diffusion: Spread of a population at time $t=20$ for species $u, v$ (Example 2).

conditions for the development of instabilities (see Section 9) are satisfied. Figure 11 displays the Turing spaces for the parameter pairs $a_{1}, a_{2} b_{1}, b_{2}$ and $c_{1}, c_{2}$. Inside the marked regions, obtained according to conditions (i) and (ii) from Section 9 , the system is expected to exhibit unstable spatial patterns.

From Figure 6 it is clearly seen that the system evolves from noise to spatial patterns qualitatively similar as those produced in the previous examples (smooth interfaces between both species). We also provide a profile view of the same simulation in Figure 7. It consists of a cut of the numerical solution at the level $y=0$ (this is, a 1D slide of the computational domain). It can be observed that even 


\begin{tabular}{|c|c|c|c|c|c|c|c|c|}
\hline & $\mathcal{N}$ & $h$ & $L^{1}$-error & $r^{1}(u)$ & $L^{1}-$ error & $r^{1}(u)$ & $L^{1}$-error & $r^{1}(u)$ \\
\hline Time & & & \multicolumn{2}{|c|}{$t=20$} & \multicolumn{2}{|c|}{$t=10$} & \multicolumn{2}{|l|}{$t=1$} \\
\hline & 1024 & $3.90 \times 10^{-3}$ & $5.68 \times 10^{-3}$ & - & $2.34 \times 10^{-3}$ & - & $9.12 \times 10^{-4}$ & - \\
\hline & 4096 & $9.77 \times 10^{-4}$ & $4.97 \times 10^{-4}$ & 1.7423 & $1.91 \times 10^{-4}$ & 1.8136 & $7.57 \times 10^{-5}$ & 1.7953 \\
\hline & 16384 & $2.44 \times 10^{-4}$ & $3.96 \times 10^{-5}$ & 1.8361 & $1.54 \times 10^{-5}$ & 1.8260 & $6.24 \times 10^{-6}$ & 1.8035 \\
\hline & 65536 & $6.10 \times 10^{-5}$ & $3.20 \times 10^{-6}$ & 1.8213 & $1.27 \times 10^{-6}$ & 1.8027 & $5.21 \times 10^{-7}$ & 1.7896 \\
\hline
\end{tabular}

TABLE 2. Number of control volumes $\mathcal{N}$, meshsize $h$, approximate $L^{1}$-errors for $u$ and observed convergence rates $r$ at different simulated times (Example 2).
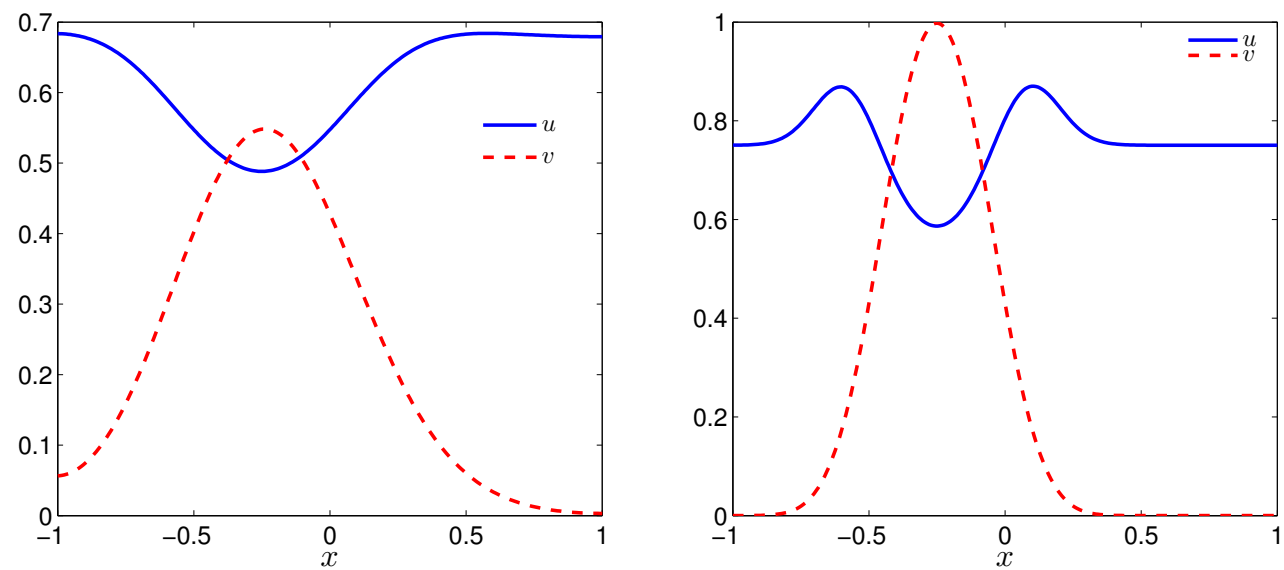

Figure 5. Profiles at time $t=20$ and $y=0$ for species $u, v$. The two cases correspond to the model without (left) and with (right) cross-diffusion (Examples 1 and 2).

when the solution seems to fall in the constant equilibrium state (at $t=0.1$ ), instabilities driven by cross-diffusion lead the solution to a stationary nonuniform spatial pattern.

\section{ON THE FORMATION OF SPATIAL PATTERNS}

The formation of spatial patterns through cross-diffusion in the long run has been addressed numerically in Section 7.3. We have shown that there exist nonuniform equilibrium solutions which exhibit spatial segregating patterns. The obtained state of coexistence is known to take place due to cross-diffusion. Here we present a brief study of the cross-diffusion driven instability of (1.1), (1.2). To this end, first note that the nontrivial constant equilibrium point of $\sqrt{1.1}),(1.2)$, i.e., the pair $\left(u^{*}, v^{*}\right)$ such that $u^{*}\left(a_{1}-b_{1} u^{*}-c_{1} v^{*}\right)=0$ and $v^{*}\left(a_{2}-b_{2} u^{*}-c_{2} v^{*}\right)=0$, is given by (see Figure 10)

$$
u^{*}=\frac{a_{2} c_{1}-a_{1} c_{2}}{b_{2} c_{1}-b_{1} c_{2}}, \quad v^{*}=\frac{a_{2} b_{1}-a_{1} b_{2}}{b_{1} c_{2}-b_{2} c_{1}} .
$$

This solution in (9.1) is positive (which means that there exists a coexistence state) if

$$
\frac{b_{1}}{b_{2}}>\frac{a_{1}}{a_{2}}>\frac{c_{1}}{c_{2}} \quad \text { or } \quad \frac{b_{1}}{b_{2}}<\frac{a_{1}}{a_{2}}<\frac{c_{1}}{c_{2}} .
$$

The state $\left(u^{*}, v^{*}\right)$ is also an equilibrium point for the following kinetic formulation associated to (1.1), (1.2):

$$
\begin{aligned}
& \partial_{t} u=u\left(a_{1}-b_{1} u-c_{1} v\right), \\
& \partial_{t} v=v\left(a_{2}-b_{2} u-c_{2} v\right), \quad(x, t) \in Q_{T}
\end{aligned}
$$



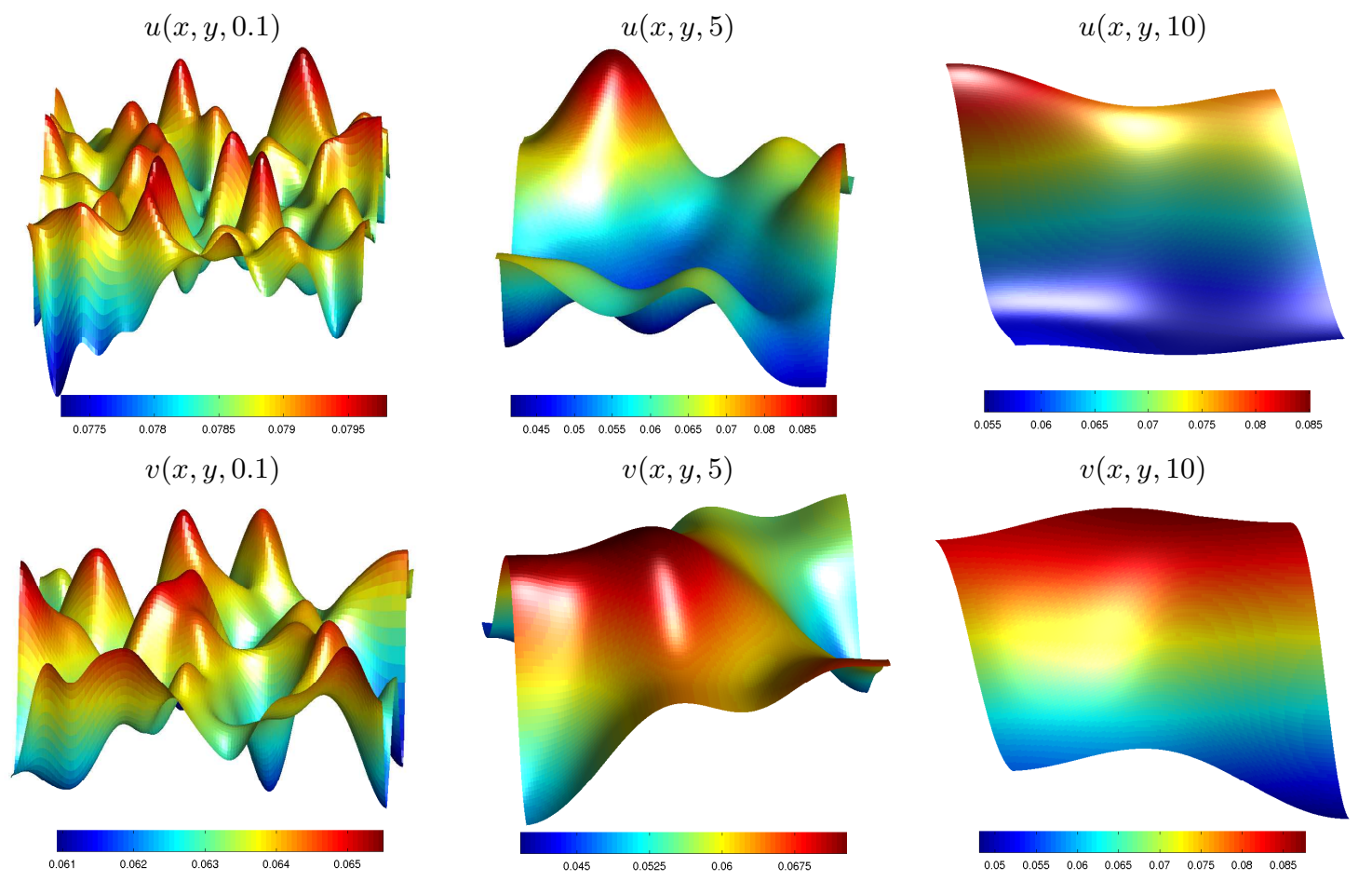

Figure 6. Model with cross-diffusion: Spread of a population at times $t=1, t=5$, and $t=10$ for species $u$ (top) and $v$ (bottom) starting from random initial data (Example 3$)$. The figure for $v(x, 10)$ has been rotated only for visualization purposes.

and $\left(u^{*}, v^{*}\right)$ is linearly stable with respect to this system if

$$
\operatorname{tr}(J)<0 \quad \text { and } \quad \operatorname{det}(J)>0
$$

where

$$
J=\left[\begin{array}{ll}
\partial_{u}\left(u\left(a_{1}-b_{1} u-c_{1} v\right)\right) & \partial_{v}\left(u\left(a_{1}-b_{1} u-c_{1} v\right)\right) \\
\partial_{u}\left(v\left(a_{2}-b_{2} u-c_{2} v\right)\right) & \partial_{v}\left(v\left(a_{2}-b_{2} u-c_{2} v\right)\right)
\end{array}\right] .
$$

This yields to the condition

$$
\frac{b_{1}}{b_{2}}>\frac{a_{1}}{a_{2}}>\frac{c_{1}}{c_{2}}
$$

Obviously, there exist other equilibrium points, namely the trivial solution $(0,0)$ and the semi-trivial solutions $\left(\frac{a_{1}}{b_{1}}, 0\right)$ and $\left(0, \frac{a_{2}}{c_{2}}\right)$, but these points are unstable with respect to the ODE $(9.2$.

For the instability of $\left(u^{*}, v^{*}\right)$ with respect to $11.1,11.2$, Tian et al. [46] recently obtained the following result:

Lemma 8.1. Suppose that $\left(u^{*}, v^{*}\right)$ is a constant equilibrium of $(9.2$ satisfying $(9.3), \operatorname{tr}(D)>0$, $\operatorname{det}(D)>0$, and $0<k_{1} \leq \mu_{m, n} \leq k_{2}$. Then $\left(u^{*}, v^{*}\right)$ is an unstable equilibrium solution with respect to $1.1,1.2$. Here

$$
D=\left[\begin{array}{ll}
\partial_{u}\left(u\left(v+\frac{1}{2} u+1\right)\right) & \partial_{v}\left(u\left(v+\frac{1}{2} u+1\right)\right) \\
\partial_{u}\left(v\left(u+\frac{1}{2} v+1\right)\right) & \partial_{v}\left(v\left(u+\frac{1}{2} v+1\right)\right)
\end{array}\right]
$$


$t=0$

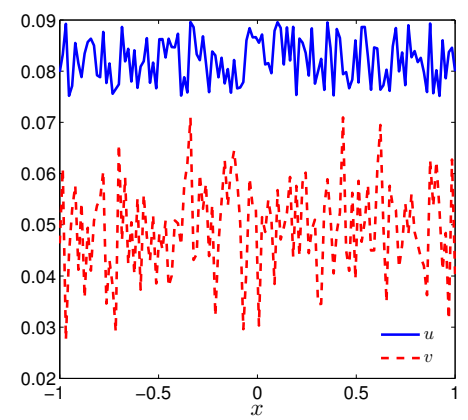

$t=5$

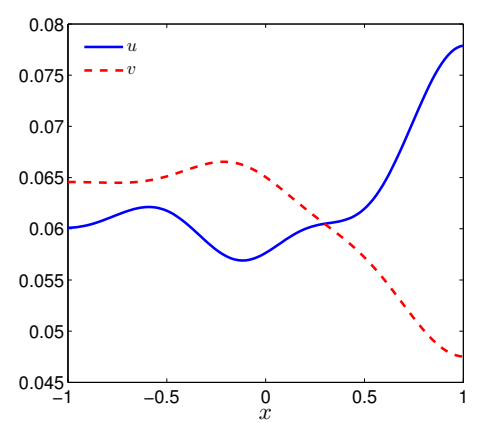

$t=0.1$
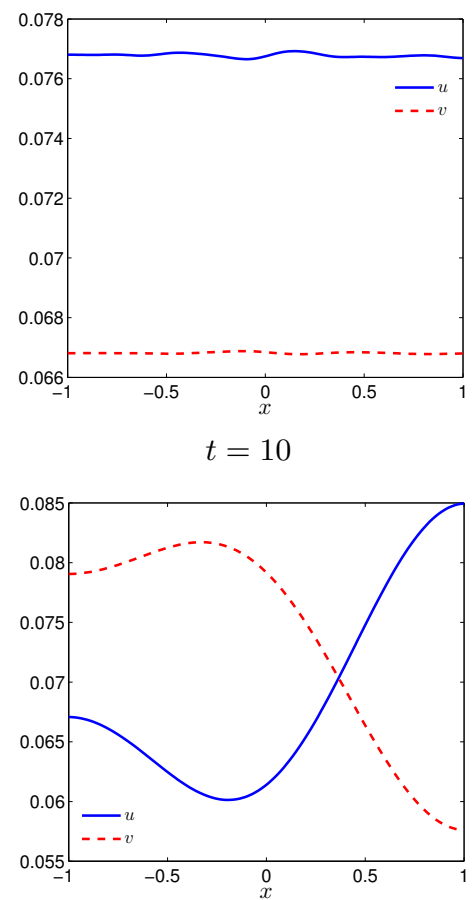

$t=1$
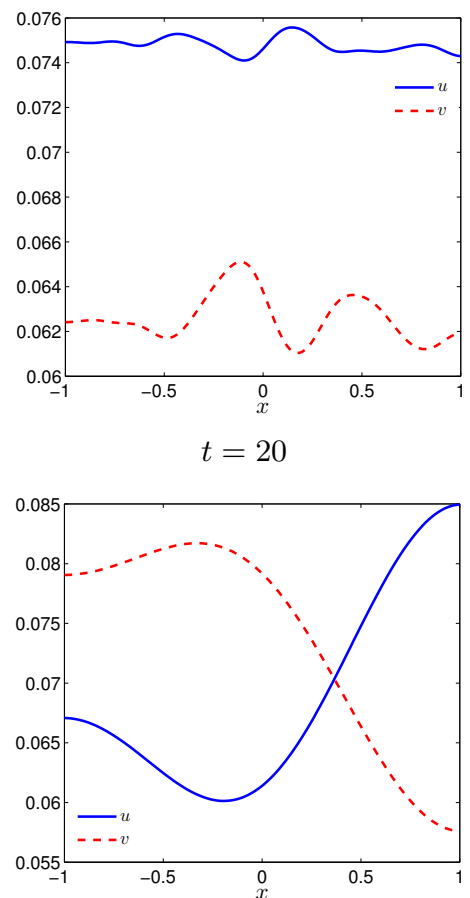

Figure 7. Model with cross-diffusion: Profile view at $y=0$ of the spread of a population at times $t=0, t=0.1, t=1, t=5, t=10$ and $t=20$ for species $u$ and $v$ (Example 3).

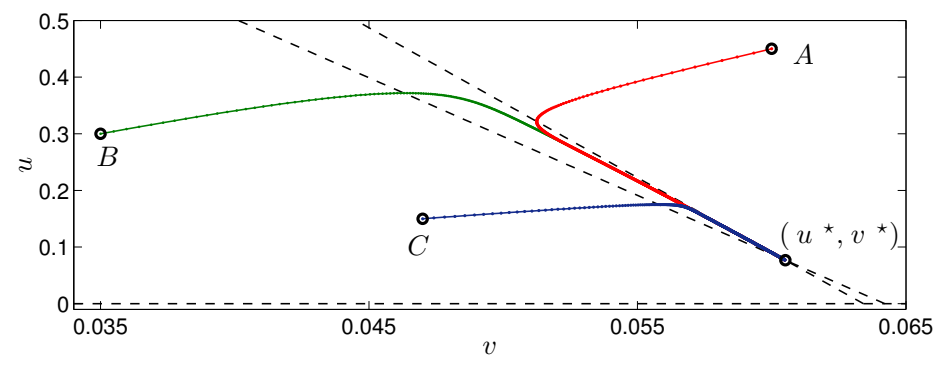

Figure 8. Phase portrait for the ODE system 9.2 . The system nullclines (in dashed lines) are the lines $u=0, v=0$ (not shown), $v=a_{1} / c_{1}-b_{1} / c_{1} u, v=a_{2} / c_{2}-b_{2} / c_{2} u$. Three computed trajectories are displayed starting from the states $A=(0.45,0.06)$, $B=(0.3,0.035)$ and $C=(0.15,0.047)$. After 500 time steps all of them reach the equilibrium point $\left(u^{*}, v^{*}\right)=(0.0769,0.0605)$. The used parameters are $a_{1}=$ $0.61 /$ year, $a_{2}=0.52 /$ year, $b_{1}=0.4575, b_{2}=0.31, c_{1}=9.5, c_{2}=8.2$.

$\mu_{m, n}=\frac{\pi^{2}}{4}(m+n)^{2}, m, n=1,2,3, \ldots$ are the eigenvalues of the Neumann problem $-\Delta w=\mu_{m, n} w$, $\partial_{n} w=0$ on the square domain $\Omega=[-1,1]^{2}$, and

$$
k_{1}=\frac{\operatorname{det}(J)+\operatorname{det}(D)-\sqrt{(-\operatorname{det}(J)-\operatorname{det}(D))^{2}-4 \operatorname{det}(D) \operatorname{det}(J)}}{2 \operatorname{det}(D)},
$$


(a)

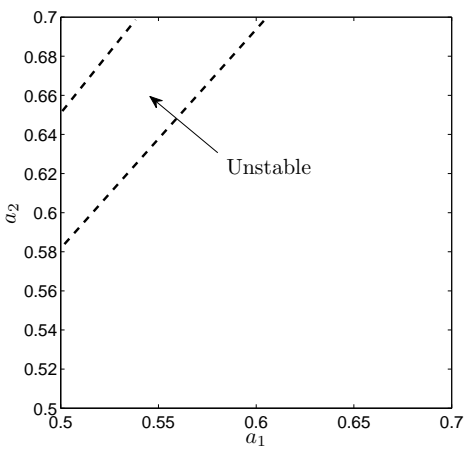

(b)

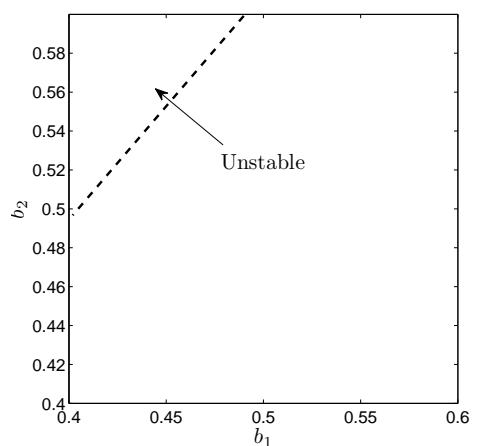

(c)

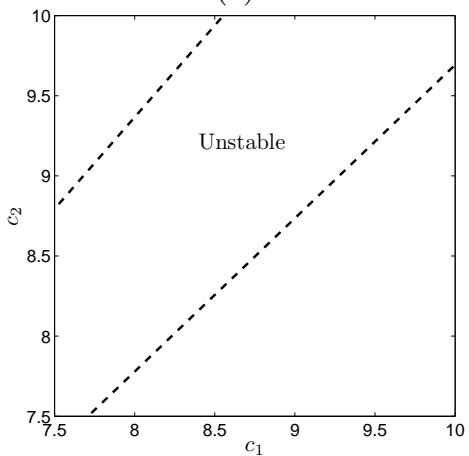

FIGURE 9. Sketched instability regions for the SKT model. The parameter spaces are determinate by fixing the remaining parameters and varying only one parameter pair: $a_{1}, a_{2}$ (a) $b_{1}, b_{2}$ (b) and $c_{1}, c_{2}$ (c) (Example 3).

$$
k_{2}=\frac{\operatorname{det}(J)+\operatorname{det}(D)+\sqrt{(-\operatorname{det}(J)-\operatorname{det}(D))^{2}-4 \operatorname{det}(D) \operatorname{det}(J)}}{2 \operatorname{det}(D)} .
$$

A further analysis, following [3, 40, 46], allows us to conclude that the fulfillment of the following conditions is sufficient for the positive equilibrium point $\left(u^{*}, v^{*}\right)$ being linearly unstable with respect to the particular case of system $11.1,(1.2)$ :

(i) $\frac{b_{1}}{b_{2}}>\frac{a_{1}}{a_{2}}>\frac{c_{1}}{c_{2}}$.

(ii) $\max \left\{\frac{c_{1}}{b_{1}}, \frac{b_{2}}{c_{2}}\right\}>\max \left\{\frac{u^{*}}{v^{*}}, \frac{v^{*}}{u^{*}}\right\}$.

Figure 11 sketches the parameter spaces where these conditions hold. The specified curves separate the regions where unstable solutions to $\sqrt{1.1},(1.2)$ arise. In Figure 11(a) the following parameters are fixed: $b_{1}=0.4575, b_{2}=0.31, c_{1}=9.5, c_{2}=8.2$, while in Figures $11(\mathrm{~b})$ and $11(\mathrm{c})$, the fixed parameters are $a_{1}=0.61 /$ year, $a_{2}=0.52 /$ year, $c_{1}=9.5, c_{2}=8.2$ and $a_{1}=0.61 /$ year, $a_{2}=0.52 /$ year, $b_{1}=$ $0.4575, b_{2}=0.31$ respectively.

\section{ON THE FORMATION OF SPATIAL PATTERNS}

The formation of spatial patterns through cross-diffusion in the long run has been addressed numerically in Section 7.3. We have shown that there exist nonuniform equilibrium solutions which exhibit spatial segregating patterns. The obtained state of coexistence is known to take place due to cross-diffusion. Here we present a brief study of the cross-diffusion driven instability of (1.1), (1.2). To this end, first note that the nontrivial constant equilibrium point of 11.1 , (1.2), i.e., the pair $\left(u^{*}, v^{*}\right)$ such that $u^{*}\left(a_{1}-b_{1} u^{*}-c_{1} v^{*}\right)=0$ and $v^{*}\left(a_{2}-b_{2} u^{*}-c_{2} v^{*}\right)=0$, is given by (see Figure 10)

$$
u^{*}=\frac{a_{2} c_{1}-a_{1} c_{2}}{b_{2} c_{1}-b_{1} c_{2}}, \quad v^{*}=\frac{a_{2} b_{1}-a_{1} b_{2}}{b_{1} c_{2}-b_{2} c_{1}} .
$$

This solution in (9.1) is positive (which means that there exists a coexistence state) if

$$
\frac{b_{1}}{b_{2}}>\frac{a_{1}}{a_{2}}>\frac{c_{1}}{c_{2}} \quad \text { or } \quad \frac{b_{1}}{b_{2}}<\frac{a_{1}}{a_{2}}<\frac{c_{1}}{c_{2}} .
$$




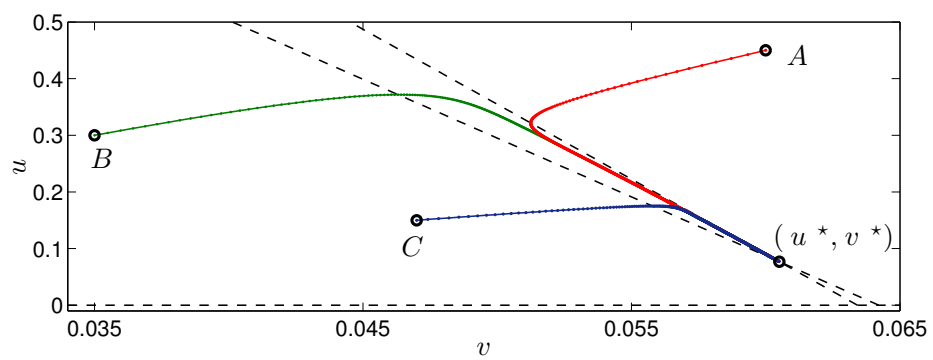

Figure 10. Phase portrait for the ODE system (9.2). The system nullclines (in dashed lines) are the lines $u=0, v=0$ (not shown), $v=a_{1} / c_{1}-b_{1} / c_{1} u, v=$ $a_{2} / c_{2}-b_{2} / c_{2} u$. Three computed trajectories are displayed starting from the states $A=(0.45,0.06), B=(0.3,0.035)$ and $C=(0.15,0.047)$. After 500 time steps all of them reach the equilibrium point $\left(u^{*}, v^{*}\right)=(0.0769,0.0605)$. The used parameters are $a_{1}=0.61 /$ year, $a_{2}=0.52 /$ year, $b_{1}=0.4575, b_{2}=0.31, c_{1}=9.5, c_{2}=8.2$.

The state $\left(u^{*}, v^{*}\right)$ is also an equilibrium point for the following kinetic formulation associated to 1.1), 1.2):

$$
\begin{aligned}
& \partial_{t} u=u\left(a_{1}-b_{1} u-c_{1} v\right), \\
& \partial_{t} v=v\left(a_{2}-b_{2} u-c_{2} v\right), \quad(x, t) \in Q_{T}
\end{aligned}
$$

and $\left(u^{*}, v^{*}\right)$ is linearly stable with respect to this system if

$$
\operatorname{tr}(J)<0 \text { and } \operatorname{det}(J)>0,
$$

where

$$
J=\left[\begin{array}{ll}
\partial_{u}\left(u\left(a_{1}-b_{1} u-c_{1} v\right)\right) & \partial_{v}\left(u\left(a_{1}-b_{1} u-c_{1} v\right)\right) \\
\partial_{u}\left(v\left(a_{2}-b_{2} u-c_{2} v\right)\right) & \partial_{v}\left(v\left(a_{2}-b_{2} u-c_{2} v\right)\right)
\end{array}\right] .
$$

This yields to the condition

$$
\frac{b_{1}}{b_{2}}>\frac{a_{1}}{a_{2}}>\frac{c_{1}}{c_{2}} .
$$

Obviously, there exist other equilibrium points, namely the trivial solution $(0,0)$ and the semi-trivial solutions $\left(\frac{a_{1}}{b_{1}}, 0\right)$ and $\left(0, \frac{a_{2}}{c_{2}}\right)$, but these points are unstable with respect to the ODE 9.2 .

For the instability of $\left(u^{*}, v^{*}\right)$ with respect to 1.1), 1.2), Tian et al. 46] recently obtained the following result:

Lemma 9.1. Suppose that $\left(u^{*}, v^{*}\right)$ is a constant equilibrium of $(9.2$ satisfying $(9.3), \operatorname{tr}(D)>0$, $\operatorname{det}(D)>0$, and $0<k_{1} \leq \mu_{l, m} \leq k_{2}$. Then $\left(u^{*}, v^{*}\right)$ is an unstable equilibrium solution with respect to (1.1), (1.2). Here

$$
D=\left[\begin{array}{ll}
\partial_{u}\left(u\left(v+\frac{1}{2} u+1\right)\right) & \partial_{v}\left(u\left(v+\frac{1}{2} u+1\right)\right) \\
\partial_{u}\left(v\left(u+\frac{1}{2} v+1\right)\right) & \partial_{v}\left(v\left(u+\frac{1}{2} v+1\right)\right)
\end{array}\right]
$$

$\mu_{l, m}=\frac{\pi^{2}}{4}(l+m)^{2}, l, m=1,2,3, \ldots$ are the eigenvalues of the Neumann problem $-\Delta w=\mu_{l, m} w$, $\partial_{n} w=0$ on the square domain $\Omega=[-1,1]^{2}$, and

$$
\begin{aligned}
& k_{1}=\frac{\operatorname{det}(J)+\operatorname{det}(D)-\sqrt{(-\operatorname{det}(J)-\operatorname{det}(D))^{2}-4 \operatorname{det}(D) \operatorname{det}(J)}}{2 \operatorname{det}(D)}, \\
& k_{2}=\frac{\operatorname{det}(J)+\operatorname{det}(D)+\sqrt{(-\operatorname{det}(J)-\operatorname{det}(D))^{2}-4 \operatorname{det}(D) \operatorname{det}(J)}}{2 \operatorname{det}(D)} .
\end{aligned}
$$


(a)

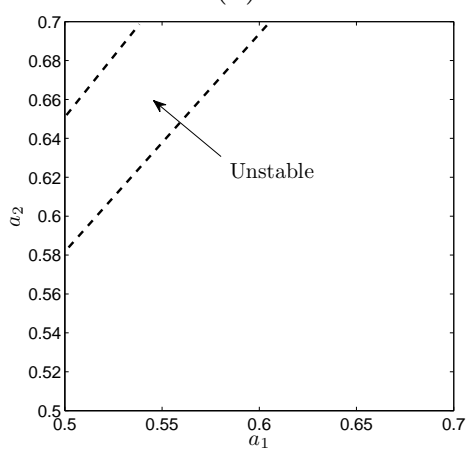

(b)

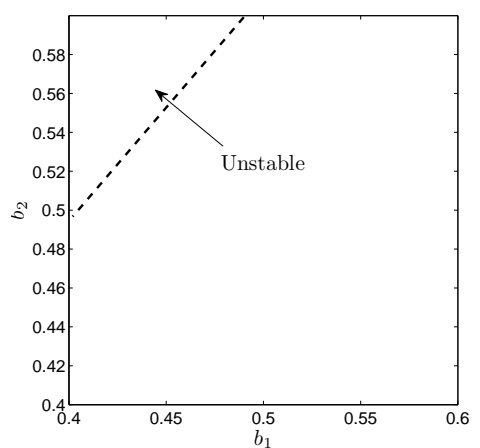

(c)

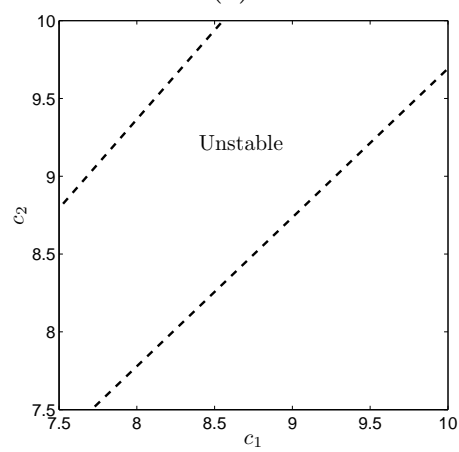

FIGURE 11. Sketched instability regions for the SKT model. The parameter spaces are determinate by fixing the remaining parameters and varying only one parameter pair: $a_{1}, a_{2}$ (a) $b_{1}, b_{2}(\mathrm{~b})$ and $c_{1}, c_{2}$ (c) (Example 3 ).

A further analysis, following [3, 40, 46, allows us to conclude that the fulfillment of the following conditions is sufficient for the positive equilibrium point $\left(u^{*}, v^{*}\right)$ being linearly unstable with respect to the particular case of system $1.1,(1.2)$ :

(i) $\frac{b_{1}}{b_{2}}>\frac{a_{1}}{a_{2}}>\frac{c_{1}}{c_{2}}$.

(ii) $\max \left\{\frac{c_{1}}{b_{1}}, \frac{b_{2}}{c_{2}}\right\}>\max \left\{\frac{u^{*}}{v^{*}}, \frac{v^{*}}{u^{*}}\right\}$.

Figure 11 sketches the parameter spaces where these conditions hold. The specified curves separate the regions where unstable solutions to 1.1$),(1.2)$ arise. In Figure 11(a) the following parameters are fixed: $b_{1}=0.4575, b_{2}=0.31, c_{1}=9.5, c_{2}=8.2$, while in Figures 11(b) and 11(c), the fixed parameters are $a_{1}=0.61 /$ year, $a_{2}=0.52 /$ year, $c_{1}=9.5, c_{2}=8.2$ and $a_{1}=0.61 /$ year, $a_{2}=0.52 /$ year, $b_{1}=$ $0.4575, b_{2}=0.31$ respectively.

\section{Appendix A. A discrete $L^{1}$ COmpaCtness lemma}

The space-time compactness lemma stated and proved in this Appendix is a result that can be used in many situations, yet in most cases simpler techniques apply. For the case of non-degenerate parabolic equations, the $L^{2}$ techniques of [20] have been successfully used for many schemes and applications. These techniques generalize to the degenerate parabolic-elliptic and parabolic-hyperbolic problems, see [22] and [23], 4, respectively. The argument of 4 is $L^{1}$-based, and it works in the case of a non-Lipschitz degeneracy. The below lemma is also $L^{1}$-based; we state it for the nondegenerate parabolic case, the adaptation to the parabolic-elliptic degeneracy of the kind considered in [22, [5] being straightforward. Notice that the arguments of the lemma cannot apply to the parabolichyperbolic problems, as the ones considered in [23], 4].

In order to state the lemma so that it apply to a large class of finite volume schemes, let us recall and somewhat enrich the notation. Indeed, the result of the lemma is valid for a wide class of finite volume discretizations of evolution equations (see Remark 9.1 below), and the proof appears simpler when the general structure of the discrete equations is exhibited.

As in Section 3. $\left(\mathcal{T}_{h}\right)_{h}$ is a family of admissible meshes of $\Omega \subset \mathbb{R}^{l}, l \geq 1$, and $\left(\Delta t_{h}\right)_{h}$ are the associated time steps; both $\Delta t_{h}$ and the size of the mesh $\mathcal{T}_{h}$ tend to zero as $h \rightarrow 0$. We retain the notation $K, x_{K}$ for the volumes and their centers, and the notation $\sigma_{K L}$ for the interface associated 
with a volume $K$ and its neighbor $L \in N(K)$. Let us mark as "boundary volumes" the volumes located adjacent to the boundary $\partial \Omega \cdot 1$

As previously, $d_{K, L}$ denotes the distance between the centers of neighbor volumes and $\eta_{K, L}$, the unit normal vector to $\sigma_{K, L}$ that points from $K$ towards $L$. In addition, we introduce the notation $\overrightarrow{\mathcal{F}}_{K, L}$ for an arbitrary $\mathbb{R}^{l}$ vector associated with the interface $\sigma_{K, L}$; we only require that $\overrightarrow{\mathcal{F}}_{K, L}=\overrightarrow{\mathcal{F}}_{L, K}$. Therefore we denote by $\mathcal{E}_{h}$ the set of all interfaces $\sigma_{K, L}=\sigma_{L, K}$ between neighbor volumes, and index the entries $\overrightarrow{\mathcal{F}}_{K, L}$ by the interfaces $\sigma_{K, L} \in \mathcal{E}_{h}$.

Further, a diamond $T_{K, L}$ is constructed upon the interface $\sigma_{K, L}$, having $x_{K}, x_{L}$ for vertices (see Figure 11). Both volumes $K$ and diamonds $T_{K, L}=T_{L, K}$ form partitions of $\Omega$ (up to a set of measure zero). Therefore we assimilate a discrete function $\left(w_{K}\right)_{K \in \mathcal{T}_{h}}$ on $\Omega$ to the piecewise constant function $w_{h}:=\sum_{K \in \mathcal{T}_{h}} w_{K} \mathbb{1}_{K}$; a discrete function $w_{h}$ is said to be null on $\partial \Omega$, if the entry $w_{K}$ is zero for all boundary volume $K$. Similarly, a discrete field $\left(\overrightarrow{\mathcal{F}}_{K, L}\right)_{\sigma_{K, L} \in \mathcal{E}_{h}}$ is assimilated to the piecewise constant vector-function

$$
\overrightarrow{\mathcal{F}}_{h}:=\sum_{\sigma_{K, L} \in \mathcal{E}_{h}} \overrightarrow{\mathcal{F}}_{K, L} \mathbb{1}_{T_{K, L}} .
$$

The $l$-dimensional (respectively, the $(l-1)$-dimensional) measures of $K, T_{K, L}$ (respectively, $\sigma_{K, L}$ ) are denoted by $|K|,\left|T_{K, L}\right|$ (respectively, by $\left.\left|\sigma_{K, L}\right|\right)$. For $\Omega^{\prime} \subset \Omega$, the localized $L^{1}$ norms of $w_{h}, \overrightarrow{\mathcal{F}}_{h}$ are defined as

$$
\left\|w_{h}\right\|_{L^{1}\left(\Omega^{\prime}\right)}:=\sum_{K \in \mathcal{T}_{h}: K \cap \overline{\Omega^{\prime}} \neq \emptyset}|K|\left|w_{K}\right|, \quad\left\|\overrightarrow{\mathcal{F}}_{h}\right\|_{L^{1}\left(\Omega^{\prime}\right)}:=\sum_{\sigma_{K, L} \in \mathcal{E}_{h}: \sigma_{K, L} \cap \overline{\Omega^{\prime}} \neq \emptyset}\left|T_{K, L}\right|\left|\overrightarrow{\mathcal{F}}_{K, L}\right| .
$$

In particular, the above norms are used for discrete gradients; indeed, for a given discrete function $w_{h}$, its discrete gradient is a certain discrete field $\overrightarrow{\mathcal{F}}_{h}=\nabla_{h} w_{h}$. In the case of classical two-point schemes on orthogonal meshes (cf. [20]) that we consider in this paper, $\nabla_{h} w_{h}$ is defined by (3.3), that is,

$$
\nabla_{K, L} w_{h}:=l \frac{w_{L}-w_{K}}{d_{K, L}} \eta_{K, L} .
$$

Remark that the local proportionality constraint (3.1) on the family of meshes $\left(\mathcal{T}_{h}\right)_{h}$ entails the following property of stability for the discrete gradient $\nabla_{h}$ of functions in $W^{1, \infty}(\Omega)$ discretized on the mesh $\mathcal{T}_{h}$ :

whenever $w_{h}=\left(w_{K}\right)_{K \in \mathcal{T}_{h}}$ with $w_{K}=\frac{1}{|K|} \int_{K} w$ for some $w \in W^{1, \infty}(\Omega)$, one has $\max _{K, L \in \mathcal{E}_{h}}\left|\nabla_{K, L} w_{h}\right| \leq C\|\nabla w\|_{\infty}$.

Finally, let us notice that for all discrete function $w_{h}$ on $\Omega$,

$$
\frac{\left|w_{K}-w_{L}\right|}{d_{K, L}} \leq C\left|\nabla_{K, L} w_{h}\right|
$$

Here and in the sequel, $C$ denotes a generic constant independent of the discretization parameter $h$ and of the discrete functions and fields considered.

Further, for a given discrete field $\overrightarrow{\mathcal{F}}_{h}$, its discrete divergence is defined as the discrete function $w_{h}=\operatorname{div}_{h} \overrightarrow{\mathcal{F}}_{h}$ with the entries

$$
\operatorname{div}_{K} \overrightarrow{\mathcal{F}}_{h}:=\frac{1}{|K|} \sum_{L \in \mathcal{N}(K)}\left|\sigma_{K, L}\right| \overrightarrow{\mathcal{F}}_{K, L} \cdot \eta_{K, L}
$$

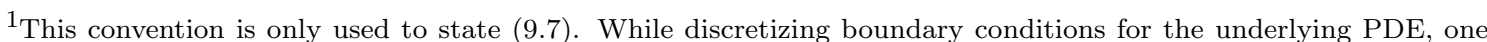
can make different choices, that are also reflected in the definition of the discrete gradient and divergence operators in volumes adjacent to the boundary. Yet, the compactness result of Lemma 9.2 being local, it does not depend on the way the boundary conditions are taken into account.
} 
A key point of the mathematical analysis of the two-point finite volume schemes on admissible meshes is the following kind of discrete duality property:

for all discrete function $w_{h}$ on $\Omega$ which is null on $\partial \Omega$, for all discrete field $\overrightarrow{\mathcal{F}}_{h}$ on $\Omega$,

$$
\sum_{K \in \mathcal{T}_{h}}|K| w_{K}\left(\operatorname{div}_{K} \overrightarrow{\mathcal{F}}_{h}\right)=\frac{1}{l} \sum_{\sigma_{K, L} \in \mathcal{E}_{h}}\left|\sigma_{K, L}\right| \overrightarrow{\mathcal{F}}_{K, L} \cdot \nabla_{K, L} w_{h} .
$$

Formula (9.6) stems from the definitions of the discrete operators $\nabla_{h}$, $\operatorname{div}_{h}$ and expresses, in a condensed way, the summation-by-parts procedure. For the purpose of proving Lemma 9.2 below, the only important consequence of the discrete duality $(9.6)$ is the following localized estimate:

$$
\begin{aligned}
& \text { for all discrete function } w_{h} \text { on } \Omega \text { which is null on } \partial \Omega \text {, for all discrete field } \overrightarrow{\mathcal{F}}_{h} \text { on } \Omega \text {, } \\
& \left|\sum_{K}\right| K\left|w_{K}\left(\operatorname{div}_{K} \overrightarrow{\mathcal{F}}_{h}\right)\right| \leq C \max _{\sigma_{K, L} \in \mathcal{E}_{h}}\left|\nabla_{K, L} w_{h}\right| \times\left\|\overrightarrow{\mathcal{F}}_{h}\right\|_{L^{1}\left(\Omega^{\prime}\right)}
\end{aligned}
$$

where $\Omega^{\prime}$ is the support of $w_{h}$

Lemma 9.2. Let $\Omega$ be an open domain in $\mathbb{R}^{l}, T>0, Q_{T}=(0, T) \times \Omega$. Let $\left(\mathcal{T}^{h}\right)_{h}$ be an admissible family of meshes of $\Omega$ satisfying the restriction $(3.1)$; let $\left(\Delta t^{h}\right)_{h}$ be the associated time steps.

For all $h>0$, assume that discrete functions $\left(u_{h}^{n+1}\right)_{n \in\left[0, N_{h}\right]},\left(f_{h}^{n+1}\right)_{n \in\left[0, N_{h}\right]}$ and discrete fields $\left(\overrightarrow{\mathcal{F}}_{h}^{n+1}\right)_{n \in\left[0, N_{h}\right]}$ satisfy the discrete evolution equations

$$
\text { for } n \in\left[0, N_{h}\right], \quad \frac{u_{h}^{n+1}-u_{h}^{n}}{\Delta t}=\operatorname{div}_{h}\left[\overrightarrow{\mathcal{F}}_{h}^{n+1}\right]+f_{h}^{n+1}
$$

with a family $\left(u_{h}^{0}\right)_{h}$ of initial data. Assume that for all $\Omega^{\prime} \Subset \Omega$, there exists a constant $M\left(\Omega^{\prime}\right)$ such that

$$
\sum_{n=0}^{N_{h}} \Delta t\left\|u_{h}^{n+1}\right\|_{L^{1}\left(\Omega^{\prime}\right)}+\sum_{n=0}^{N_{h}} \Delta t\left\|f_{h}^{n+1}\right\|_{L^{1}\left(\Omega^{\prime}\right)}+\sum_{n=0}^{N_{h}} \Delta t\left\|\overrightarrow{\mathcal{F}}_{h}^{n+1}\right\|_{L^{1}\left(\Omega^{\prime}\right)} \leq M\left(\Omega^{\prime}\right) .
$$

and, moreover,

$$
\sum_{n=0}^{N_{h}} \Delta t\left\|\nabla_{h} u_{h}^{n+1}\right\|_{L^{1}\left(\Omega^{\prime}\right)} \leq M\left(\Omega^{\prime}\right)
$$

Assume that the family $\left(u_{0}^{h}\right)_{h}$ is bounded in $L_{l o c}^{1}(\Omega)$. Then there exists a measurable function $u$ on $Q_{T}$ such that, along a subsequence,

$$
\sum_{n=0}^{N_{h}} \sum_{K \in \mathcal{T}_{h}} u_{K}^{n+1} \mathbb{1}_{\left(t^{n}, t^{n+1}\right] \times K} \longrightarrow u \quad \text { in } L_{l o c}^{1}([0, T] \times \Omega) \text { as } h \rightarrow 0 .
$$

Remark 9.1. (i) The assumption on $\left(u_{0}^{h}\right)_{h}$ can be bypassed easily, but it is not restrictive in practice. (ii) The proof of the lemma does not use the specific structure of the space mesh and of the discrete gradient and divergence operators, but only the three properties (9.4), (9.5) and (9.7) that are shared by many known schemes. Therefore the lemma is not restricted to the finite volume schemes on admissible (i.e., orthogonal) meshes with the two-point gradient reconstruction, as considered in this paper. For instance, for the so-called DDFV schemes (see Hermeline [31, Domelevo and Omnès [18, Andreianov, Bendahmane and Karlsen [4] and references therein) or the "complementary volumes" schemes such as those of Afif and Amaziane [1] and Handlovičová, Mikula and Sgallari [30, the lemma can be stated in exactly the same way.

(iii) As it is stated, the lemma is a local result and does not depend of the boundary conditions for the scheme. Clearly, the compactness of the family of discrete solutions in the sense of the a.e. convergence follows by the diagonal extraction argument. Moreover, if a uniform up-to-the-boundary $L^{p}$ bound on the discrete solutions is given, the strong $L^{q}\left(Q_{T}\right)$ convergence (along a subsequence) follows by the Vitali theorem, for all $q<p$. 
Proof. The proof of Lemma 9.2 is divided into four steps, the heart of it being Step 3.

We will denote by $u^{h}(t, x)$ the discrete solution $\sum_{n=0}^{N_{h}} u_{h}^{n+1}(x) \mathbb{1}_{\left(t^{n}, t^{n+1}\right]}(t)$; similarly, we introduce the functions $f^{h}$ and $\overrightarrow{\mathcal{F}}^{h}$ in $L_{l o c}^{1}\left(Q_{T}\right)$.

Step 1: Assumption 9.5) and the uniform estimate 9.10) of the discrete gradient imply the uniform local estimate of the space translates of $u^{h}$ :

$$
\sup _{|d x| \leq \Delta} \int_{0}^{T} \int_{\Omega^{\prime}}\left|u^{h}(t, x+d x)-u^{h}(t, x)\right| d x d t \leq \Delta C M\left(\Omega_{\Delta}^{\prime}\right)
$$

of space translates of $u^{h}$. Here $\Omega_{\Delta}^{\prime}:=\left\{x \in \Omega\left|\operatorname{dist}\left(x, \Omega^{\prime}\right)<\right| \Delta \mid\right\}$, and $\Delta$ is assumed to be smaller than the distance between $\partial \Omega$ and $\overline{\Omega^{\prime}}$.

The technique of the proof of (9.11) is standard, following Eymard, Gallouët and Herbin [20; we give it here for the sake of completeness. Notice that we only need $L_{l o c}^{1}$ translations, so that no geometric restriction on the mesh is needed (cf. the argument in Appendix of the Ref. 24], recalled in Appendix B of the present paper).

Denote by $\mathcal{V}\left(\Omega^{\prime}, \Delta\right)$ the set of all interfaces $\sigma_{K, L}$ of the mesh $\mathcal{T}$ such that $\sigma_{K, L} \cap \overline{\Omega_{\Delta}^{\prime}} \neq \emptyset$. For $x \in \Omega^{\prime}$, set $\bar{\psi}_{K, L}(x)=1$, in case the segment $[x, x+d x]$ crosses $\sigma_{K, L}$, and $\bar{\psi}_{K, L}(x)=0$ otherwise. Clearly, $\bar{\psi}_{K, L} \equiv 0$ if $x \notin \Omega_{\Delta}^{\prime}$. Also note that $\int_{\Omega^{\prime}} \bar{\psi}_{K, L}(x) d x \leq\left|\sigma_{K, L}\right| \Delta$. We have

$$
\begin{aligned}
\int_{0}^{T} \int_{\Omega^{\prime}}\left|u^{h}(t, x)-u^{h}(t, x+d x)\right| d x d t \leq \sum_{n=0}^{N_{h}} \sum_{\sigma_{K, L} \in \mathcal{E}_{h}} \Delta t_{h}\left|u_{K}^{n+1}-u_{L}^{n+1}\right| \int_{\Omega^{\prime} \Delta} \bar{\psi}_{K, L}(x) d x \\
\quad \leq \Delta \sum_{n=0}^{N_{h}} \sum_{\sigma_{K, L} \in \mathcal{V}\left(\Omega^{\prime}, \Delta\right)} \Delta t_{h}\left|\sigma_{K, L}\right|\left|u_{K}^{n+1}-u_{L}^{n+1}\right| \leq C \Delta \sum_{n=0}^{N_{h}} \sum_{\sigma_{K, L} \in \mathcal{V}\left(\Omega^{\prime}, \Delta\right)} \Delta t_{h}\left|\sigma_{K, L}\right| d_{K, L}\left|\nabla_{K, L} u^{h}\right|,
\end{aligned}
$$

where we have used 9.5 at the last step. The right-hand side of the above inequality is exactly

$$
C l \Delta \sum_{n=0}^{N_{h}}\left\|\nabla_{h} u^{h, n}\right\|_{L^{1}\left(\Omega_{\Delta}^{\prime}\right)},
$$

and we conclude using 9.10 .

Step 2: We replace the study of discrete functions $u^{h}$ (constant per cylinder $Q_{K}^{n}$ ) by the study of functions $\bar{u}^{h}$ piecewise continuous in $t$ for all $x$, constant in $x$ for all volume $K$, defined as

$$
\bar{u}^{h}(t, x)=\sum_{n=0}^{N_{h}} \sum_{K \in \mathcal{T}_{h}} \frac{1}{\Delta t_{h}}\left(\left(t-n \Delta t_{h}\right) u_{K}^{n+1}+\left((n+1) \Delta t_{h}-t\right) u_{K}^{n}\right) \mathbb{1}_{Q_{K}^{n}}(t, x) .
$$

We also extend $\bar{u}^{h}$ by the constant in time value $u^{h, N_{h}+1}$ on $\left[\Delta t\left(N_{h}+1\right),+\infty\right)$; as to $\overrightarrow{\mathcal{F}}^{h}$ and $f^{h}$, they are extended by zero values for $t>\Delta t\left(N_{h}+1\right)$. The above definitions permit us to rewrite equation 9.8 under the form

$$
\frac{\partial}{\partial t} \bar{u}^{h}=\operatorname{div}_{h} \overrightarrow{\mathcal{F}}^{h}+f^{h}
$$

where the equation is satisfied in $W^{1,1}\left(\mathbb{R}^{+}\right)$in time, for a.e. $x \in \Omega$.

In addition, we have

$$
\int_{0}^{+\infty} \int_{\Omega^{\prime}}\left|\bar{u}^{h}(t, x+d x)-\bar{u}^{h}(t, x)\right| d x d t \leq 2 \int_{0}^{T} \int_{\Omega^{\prime}}\left|u^{h}(t, x+d x)-u^{h}(t, x)\right| d x d t+2 \Delta t_{h} \int_{\Omega_{\Delta}^{\prime}}\left|u_{0}^{h}(x)\right| d x .
$$

By the result of Step 1, the assumption $\Delta t_{h} \rightarrow 0$ as $h \rightarrow 0$ and the boundedness of $\left(u_{0}^{h}\right)_{h}$ in $L^{1}\left(\Omega_{\Delta}^{\prime}\right)$, the space translates of $\bar{u}^{h}$ on $\Omega^{\prime}$ are estimated uniformly for all sequence $\left(h_{i}\right)_{i}$ convergent to zero. 
The boundedness of $\left(\bar{u}^{h_{i}}\right)_{i}$ in $L_{l o c}^{1}([0, T] \times \Omega)$ is shown in the same way. In the sequel, we drop the subscript $i$ in the notation.

Step 3 : Now we adapt the idea of Lemma 2.3 . We show that, provided $u^{h}$ solves a discrete evolution equation of the form (9.12) with terms bounded in $L_{l o c}^{1}$ and the estimate 9.11) of the space translates of $\left(\bar{u}^{h}\right)_{h}$ is available, there is also a uniform estimate of the time translates of $\left(\bar{u}^{h}\right)_{h}$ :

$$
\text { for all } \Delta \in(0, \tau], \quad \int_{0}^{+\infty} \int_{\Omega}\left|\bar{u}^{h}(t+\Delta, x)-\bar{u}^{h}(t, x)\right| d x d t \leq \widetilde{\omega}(\tau)
$$

uniformly in $h$. Here $\widetilde{\omega}: \mathbb{R}^{+} \longrightarrow \mathbb{R}^{+}$is a modulus of continuity, i.e., $\lim _{\tau \rightarrow 0} \widetilde{\omega}(\tau)=0$.

Let us construct $\widetilde{\omega}(\cdot)$ verifying $(9.13)$. First fix $h$ and fix $\Delta \in(0, \tau]$. Denote by $I^{h}(\Delta)$ the left-hand side of 9.13). For $t \geq 0$, set $w^{h}(t, \cdot)=\bar{u}^{h}(t+\Delta, \cdot)-\bar{u}^{h}(t, \cdot)$. Notice that $w^{h}(t, \cdot) \equiv 0$ for large $t$.

Take a standard family $\left(\rho_{\delta}\right)_{\delta}$ of mollifiers on $\mathbb{R}^{l}$ defined as $\rho_{\delta}(x):=\delta^{-l} \rho(x / \delta)$, where $\rho$ is a Lipschitz continuous, nonnegative function supported in the unit ball of $\mathbb{R}^{l}$, and $\int_{\mathbb{R}^{l}} \rho(x) d x=1$. In particular, we have

$$
\left|\nabla \rho_{\delta}\right| \leq \frac{C}{\delta^{l+1}} .
$$

Here and throughout the proof, $C$ will denote a generic constant independent of $h$ and $\delta$. For all $t>0$, define the function $\varphi(t, \cdot): \mathbb{R}^{l} \longrightarrow \mathbb{R}$ by $\varphi(t):=\rho_{\delta} *\left(\operatorname{sign} w^{h}(t) \mathbb{1}_{\Omega^{\prime}}\right)$. In order to lighten the notation, we do not stress the dependence of $\varphi$ on $h$ and $\delta$. Discretize $\varphi(t, \cdot)$ on the mesh $\mathcal{T}_{h}$ by setting $\varphi_{K}(t)=\frac{1}{|K|} \int_{K} \varphi(t, x) d x$; we denote $\varphi^{h}(t)$ the corresponding discrete function. Denote $\operatorname{size}\left(\mathcal{T}_{h}\right):=\max _{K \in \mathcal{T}_{h}} \operatorname{diam}(K)$. By the definition of $\varphi(t, \cdot)$, the discrete function $\varphi^{h}(t)$ is null on the set $\left\{x \in \Omega \mid \operatorname{dist}\left(x, \overline{\Omega^{\prime}}\right) \geq \delta+\operatorname{size}\left(\mathcal{T}_{h}\right)\right\}$, for all $t$. Thus for all sufficiently small $h$ and $\delta$, the support of $\varphi^{h}(t)$ is included in some domain $\Omega^{\prime \prime}, \Omega^{\prime \prime} \Subset \Omega$.

Now for for all $x \in K$, we multiply equation $(9.12)$ by $|K| \varphi(t)_{K}$, integrate in $t$ on $[s, s+\Delta]$, and make the summation over all $K$. Finally, we integrate the obtained equality in $s$ over $\mathbb{R}^{+}$to get

$$
\int_{0}^{+\infty} \sum_{K}|K| \varphi_{K}(s) w_{K}(s) d s=\int_{0}^{+\infty} \int_{s}^{s+\Delta} \sum_{K}|K| \varphi_{K}(t)\left(\operatorname{div}_{K}\left[\overrightarrow{\mathcal{F}}^{h}(t)\right]+\left(f^{h}(t)\right)_{K}\right) d t d s .
$$

Denote by $I_{\delta}^{h}(\Delta)$ the left-hand side of (9.14). Denote $Q^{\prime \prime}=\left(0,\left(N_{h}+1\right) \Delta t\right) \times \Omega^{\prime \prime}$. Using hypothesis (9.7), the definitions of discrete norms and the Fubini theorem, we infer

$$
I_{\delta}^{h}(\Delta) \leq C \Delta\left(\left\|\overrightarrow{\mathcal{F}}^{h}\right\|_{L^{1}\left(Q^{\prime \prime}\right)} \max _{t>0} \max _{\sigma_{K, L}}\left|\nabla_{K, L} \varphi^{h}(t)\right|+\left\|\varphi^{h}\right\|_{L^{\infty}\left(Q^{\prime \prime}\right)}\left\|f^{h}\right\|_{L^{1}\left(Q^{\prime \prime}\right)}\right) .
$$

Now the the $L_{l o c}^{1}([0, T] \times \Omega)$ bounds $(9.9)$ on $\left(\overrightarrow{\mathcal{F}}^{h}\right)_{h},\left(f^{h}\right)_{h}$, the bounds $|\varphi(t, \cdot)| \leq 1,|\nabla \varphi(t, \cdot)| \leq C / \delta^{l+1}$, and assumption $(9.4$ yield the estimate

$$
I_{\delta}^{h}(\Delta) \leq C \Delta\left(1+\delta^{-l-1}\right)
$$

for all $h$ and $\delta$ small enough, uniformly in $h$. Now, notice that by the definition of $\varphi_{K}(t)$,

$|K|\left(\left|w_{K}(t)\right|-w_{K}(t) \varphi_{K}(t)\right)=|K|\left|w^{h}(t, x)\right|-w_{K}(t) \int_{K} \varphi(t, x) d x=\int_{K}\left(\left|w^{h}(t, x)\right|-w^{h}(t, x) \varphi(t, x)\right) d x ;$ therefore

$$
I^{h}(\Delta)-I_{\delta}^{h}(\Delta)=\int_{0}^{+\infty} \int_{\Omega}\left(\left|w^{h}(t, x)\right|-w^{h}(t, x) \varphi(t, x)\right) d x d t .
$$

Starting from this point, the argument of Kruzhkov [34 applies exactly as for the "continuous" case of Lemma 2.3 Set $U_{\delta}^{\prime}:=\left\{x \in \mathbb{R}^{l} \mid \operatorname{dist}\left(x, \partial \Omega^{\prime}\right)<\delta\right\}$; notice that $U_{\delta}^{\prime} \subset \Omega^{\prime \prime} \Subset \Omega$ for all $\delta$ small enough. Notice that without loss of restriction, the boundary of $\Omega^{\prime}$ can be chosen regular enough so that to ensure that meas $\left(U_{\delta}^{\prime}\right)$ goes to zero as $\delta \rightarrow 0$. By the result of Step 1 of the lemma and the Frechet-Kolmogorov theorem, the family $\left(\int_{0}^{+\infty}\left|w^{h}(t, \cdot)\right| d t\right)_{h}$ is relatively compact in $L_{l o c}^{1}(\Omega)$. 
Therefore these functions are equi-integrable on $\Omega^{\prime \prime}$, so that $\int_{0}^{+\infty} \int_{U_{\delta}^{\prime}}\left|w^{h}(t, x)\right| d x d t \leq \hat{\omega}(\delta)$ uniformly in $h$, with $\lim _{\delta \rightarrow 0} \hat{\omega}(\delta)=0$. Now by the definition of $\varphi$, from formula (9.16) we deduce that

$$
\left|I^{h}(\Delta)-I_{\delta}^{h}(\Delta)\right| \leq 2 \int_{0}^{+\infty} \int_{U_{\delta}^{\prime}}\left|w^{h}(t, x)\right| d x d t+\int_{0}^{+\infty} \int_{\Omega^{\prime} \backslash U_{\delta}^{\prime}}\left|w^{h}(t, x)\right|-w^{h}(t, x)\left(\rho_{\delta} * \operatorname{sign} w^{h}(t)\right)(x) \mid d x d t ;
$$

the first term in the right-hand side accounts for the action of the truncation $\mathbb{1}_{\Omega^{\prime}}$ in the definition of $\varphi$. Using the standard properties of $\rho_{\delta}$, we infer

$$
\left|I^{h}(\Delta)-I_{\delta}^{h}(\Delta)\right| \leq 2 \hat{\omega}(\delta)+\int_{0}^{+\infty} \int_{\Omega^{\prime} \backslash U_{\delta}^{\prime}} \int_{\mathbb{R}^{l}} \rho_{\delta}(x-y)|| w^{h}(t, x)\left|-w^{h}(t, x) \operatorname{sign} w^{h}(t, y)\right| d y d x d t .
$$

Now note the key inequality:

$$
\forall a, b \in \mathbb{R} \quad|| a|-a \operatorname{sign} b| \leq 2|a-b| .
$$

Setting $\sigma:=(x-y) / \delta$, we infer

$$
\begin{aligned}
& \left|I^{h}(\Delta)-I_{\delta}^{h}(\Delta)\right| \leq 2 \hat{\omega}(\delta)+2 \int_{0}^{+\infty} \int_{\Omega^{\prime}} \int_{\mathbb{R}^{l}} \rho_{\delta}(x-y)\left|w^{h}(t, x)-w^{h}(t, y)\right| d y d x d t \leq \\
& \quad \leq 2 \hat{\omega}(\delta)+2 \int_{\mathbb{R}^{l}} \rho(\sigma) \int_{0}^{+\infty} \int_{\Omega^{\prime}}\left|\bar{u}^{h}(t, x)-\bar{u}^{h}(t, x-\delta \sigma)\right| d x d t d \sigma \leq 2 \hat{\omega}(\delta)+2 \omega(\delta),
\end{aligned}
$$

where $\omega(\cdot)$ is the modulus of continuity controlling the space translates of $\bar{u}^{h}$ in $\Omega^{\prime}$. Indeed, by Steps 1 and 2 of the proof, one can choose $\omega(\cdot)$ independent of $h$. Combining 9.15) with 9.17), we conclude that the function

$$
\widetilde{\omega}(\tau):=\inf _{\delta>0} C\left\{\tau\left(1+\delta^{-l-1}\right)+2 \hat{\omega}(\delta)+2 \omega(\delta)\right\}
$$

upper bounds the quantity $I^{h}$. Because $\widetilde{\omega}(\tau)$ tends to 0 as $\tau \rightarrow 0$, this proves 9.13.

Step 4 : By the Riesz-Frechet-Kolmogorov compactness criterion, the relative compactness of $\left(\bar{u}^{h}\right)_{h}$ in $L_{l o c}^{1}([0, T] \times \Omega)$ is a consequence of the estimates of Steps 2 and 3 . In order to conclude, it suffices to show that $\left\|u^{h}-\bar{u}^{h}\right\|_{L^{1}\left(\Omega^{\prime}\right)} \rightarrow 0$ as $h \rightarrow 0$.

An easy calculation shows that for all $a, b \in \mathbb{R}, \int_{0}^{1}|\theta a+(1-\theta) b| d \theta \geq \frac{1}{2}(|a|+|b|)$. Applying this inequality to $a=u_{h}^{(n+1)}-u_{h}^{n}, b=u_{h}^{n}-u_{h}^{(n-1)}$, from the definition of $\bar{u}^{h}$ we deduce

$$
\int_{0}^{T} \int_{\Omega^{\prime}}\left|u^{h}(t, x)-\bar{u}^{h}(t, x)\right| d x d t \leq 2 \int_{0}^{T+\Delta t_{h}} \int_{\Omega^{\prime}}\left|\bar{u}^{h}\left(t+\Delta t_{h}, x\right)-\bar{u}^{h}(t, x)\right| d x d t .
$$

Since $\Delta t_{h}$ tends to zero as $h \rightarrow 0$, estimate (9.13) of Step 3 implies that the right-hand side of the above inequality converges to zero as $h$ tends to zero. This ends the proof of the lemma.

\section{Appendix B. Discrete Sobolev embedding inequalities.}

Under different assumptions, discrete Sobolev embeddings with Neumann boundary conditions in the case $p=2$ was recently derived by Chainais-Hillairet and Droniou in 11] (see also Filbet 25]) and by Glitzky and Griepentrog [29. Here we give a straightforward adaptation to the case of Neumann or mixed boundary conditions of the proof of Eymard, Gallouët, Herbin (see the appendix of 24]), initially written for the Dirichlet case. We look at a slightly more general finite volume meshes than those considered in the main part of the paper.

We consider an open polygonal domain $\Omega$ of $\mathbb{R}^{d}, d \geq 2$. A mesh $\mathcal{T}$ of $\Omega$ is a finite volume mesh in the sense of 20, 24] (without the orthogonality condition on the mesh) and with the two following admissibility conditions:

For all neighbor volumes $K, L$, the angle $\alpha_{K, L}$ between $\overrightarrow{x_{K} x_{L}}$ and the interface $\sigma_{K, L}$

is separated from 0 and $\pi$, meaning that $\operatorname{reg}(\mathcal{T}) \cos \alpha_{K, L} \geq 1$ 
(this condition replaces the mesh orthogonality assumption), and

$$
\text { For all volume } K \text { and interface } \sigma_{K, L},\left|\sigma_{K, L}\right| d_{K, L} \leq \operatorname{reg}(\mathcal{T})|K| \text {. }
$$

Here $\operatorname{reg}(\mathcal{T})$ is a constant measuring the distortion of the mesh.

It is evident that the latter condition can replace the usual "commeasurability" assumption on the two parts $d_{K, \sigma}$ and $d_{L, \sigma}$ of $d_{K, L}$, used in [20, 24]. Notice that the assumption (9.19) even allows $x_{K}$ to leave $K$ (as it happens, e.g., if one takes for $K$ a triangle with an angle greater than or equal to $\pi / 2$, and picks for the center $x_{K}$ of $K$ the point equi-distant of the vertices of $K$ ). Notice that for the Poincaré inequality, even $(9.19)$ can be relaxed (see the proof in $[5])^{2}$, actually one merely needs $\left|\sigma_{K, L}\right| d_{K, L} \leq \operatorname{reg}(\mathcal{T}) \max \{|K|,|L|\}$. For symplectic meshes with the choice

$$
x_{K}=\text { the point equi-distant from the vertices of } K \text {, }
$$

the latter assumption is ensured by the usual Delaunay condition.

A discrete function on $\mathcal{T}$ is the piecewise constant function

$$
u(x)=\sum_{K \in h} u_{K} \mathbb{1}_{K}(x) ;
$$

the $L^{p}$ norm of its discrete gradient can be defined as

$$
\left\|\nabla_{h} u\right\|_{L^{p}(\Omega)}:=\sum_{K, L \in \mathcal{E}}\left|\sigma_{K, L}\right| d_{K, L}\left|\frac{u_{L}-u_{K}}{d_{K, L}}\right|^{p},
$$

where the summation runs on the set $\mathcal{E}$ of all interfaces between neighbor volumes. Notice that this definition is most accurate for the case of meshes with the orthogonality condition. In general, a more natural definition would involve a constant $C(\operatorname{reg}(\mathcal{T}))$ in the left-hand side related to assumption (9.18.

The following result states the Sobolev embedding inequalities for discrete functions with control by a mean value (either inside the domain, or on a part of the boundary). These inequalities are useful for study of PDEs with, respectively, Neumann boundary conditions and mixed non homogeneous Dirichlet/Neumann boundary conditions. The compactness of the subcritical embeddings is an easy consequence. The key assumption we make is that the corresponding Poincaré inequality is already known. This is the case if $p=2$; we refer to Coudière, Gallouët and Herbin [17] (see also [20]) for $d=2,3$ and to Glitzky and Griepentrog 29] for an argument that allows for general $d$. The proofs can be adapted to the case $p \neq 2$. Notice that the assumption 9.19 is enough for these proofs to work.

Proposition 9.3. Let $u$ be a discrete function on the mesh $\mathcal{T}, \omega \subset \Omega$ be a fixed open set and $\Gamma \subset \partial \Omega$ be a fixed part of the boundary with positive $(d-1)$-dimensional measure. Set

$$
\text { either } \quad m_{u}:=\frac{1}{|\omega|} \int_{\omega} u, \quad \text { or } \quad m_{u}:=\frac{1}{|\Gamma|} \int_{\Gamma} u
$$

(with $|\cdot|$ representing the $d$ and the $(d-1)$-dimensional Lebesgue measures, respectively). Assume that the following Poincaré inequality holds:

$$
\left\|u-m_{u}\right\|_{L^{p}(\Omega)} \leq C(\Omega, d, p, \omega, \Gamma, \operatorname{reg}(\mathcal{T}))\left\|\nabla_{h} u\right\|_{L^{p}(\Omega)},
$$

where $\operatorname{reg}(\mathcal{T})$ is the regularity constant of the mesh in the sense of $(9.18)$ and $(9.19)$.

Then also the Sobolev embedding inequalities hold:

$$
\|u\|_{L^{q}(\Omega)} \leq C(\Omega, p, q, \omega, \Gamma, \operatorname{reg}(\mathcal{T}))\left(\left\|\nabla_{h} u\right\|_{L^{p}(\Omega)}+m_{u}\right)
$$

for all $q \in\left[1, p^{*}\right]$ if $p<d, p^{*}=\frac{p d}{d-p}$, and for all $q \in[1,+\infty)$ if $p \geq d$.

\footnotetext{
2 actually, the hint of [5] also gives the discrete embedding of $W^{1, p}$ into $L^{p \frac{d}{d-1}}=L^{p 1^{*}}$ but one cannot attain exponents $q \in\left(p 1^{*}, p^{*}\right]$ with the same technique.
} 
The proof is based upon the following " $B V$ version" of the $W^{1,1}$ compactness for discrete functions:

Lemma 9.4. For all discrete function $u$ on a polygonal partition $\mathcal{T}$ of $\Omega$ satisfying (9.18),

$$
\left\|u-\frac{1}{|\Omega|} \int_{\Omega} u\right\|_{L^{1^{*}}(\Omega)} \leq C(\Omega, \operatorname{reg}(\mathcal{T}))\left\|\nabla_{h} u\right\|_{L^{1}(\Omega)},
$$

where $1^{*}:=\frac{d}{d-1}$.

Lemma 9.4 is deduced from the classical critical embedding inequality for $W^{1,1}(\Omega)$ functions. In the first step, the variation on $\Omega$ of a discrete function $u$ is controlled by

$$
\sum_{K, L \in \mathcal{E}}\left|\sigma_{K, L}\right|\left|u_{L}-u_{K}\right| \equiv\left\|\nabla_{h} u\right\|_{L^{1}(\Omega)}
$$

(see Lemma 5.1 in 24$]$ ). Then $u$ is extended in a small neighbourhood of $\Omega$ with a control on the variation; because $\Omega$ is polygonal, its boundary $\partial \Omega$ is Lipschitz, and we can use a partition of unity on the boundary, rectification and reflexion technique (see e.g. 19]) in order to construct the desired extension of $u$. Finally, the regularization technique by convolution permits to go from the $W^{1,1}(\Omega)$ functions to the $B V$ functions (see the proof of Lemma 5.1 in [24]).

From Proposition 9.3 we readily get

Corollary 9.5. Let $\left(u_{h}\right)_{h}$ be a family of discrete functions on meshes with uniformly bounded regularity constant $\operatorname{reg}(\mathcal{T})$. Assume that $m_{u_{h}}$ and $\left\|\nabla_{h} u_{h}\right\|_{L^{p}(\Omega)}$ are uniformly bounded. Then the family $\left(u_{h}\right)_{h}$ is relatively compact in $L^{q}(\Omega)$ for all $q<+\infty$ (if $p \geq d$ ) and for all $q<p^{*}$ (if $p<d$ ).

Indeed, as indicated in the proof of Lemma 9.4 , the $L^{p}$ bound of the discrete gradients $\nabla_{h} u_{h}$ also yields the $B V$ bound on $u_{h}$. Then (using the uniform $L^{1}$ bound on $u_{h}$ coming from Proposition 9.3p by the Helly theorem we deduce that the family $\left(u_{h}\right)_{h}$ is relatively compact in $L^{1}(\Omega)$. Then using the interpolation of Lebesgue spaces and the uniform bounds of Proposition 9.3 , we prove the claim of Corollary 9.5 .

Now we turn to the proof of the Sobolev embedding inequalities of Proposition 9.3 The proof uses a bootstrap argument in $q$ starting at $q=p$ (the Poincaré case); the technique is the one of Lemma 5.2 in [24], which in turn follows the original proof of L. Nirenberg.

Proof. Thanks to the Hölder inequality, without loss of generality we may assume that $p<d$; thus $p^{*}<+\infty$.

In the first step, we notice that 9.21) with $q=p$ follows from the Poincaré inequality 9.20 . In particular, we derive the bound

$$
\frac{1}{|\Omega|} \int_{\Omega}|u|^{\alpha} \leq C(\Omega, d, p, \omega, \Gamma, \operatorname{reg}(\mathcal{T}))\left(\left(\left\|\nabla_{h} u\right\|_{L^{p}(\Omega)}\right)^{\alpha}+\left(m_{u}\right)^{\alpha}\right)
$$

with, for the time being, $\alpha=p$.

Then we apply Lemma 9.4 to the discrete function $v:=|u|^{\alpha}$ with $\alpha=p$. As in the proof of Lemma 5.2 of [24], using the fact that

$$
\left|\frac{\left|u_{L}\right|^{\alpha}-\left|u_{K}\right|^{\alpha}}{d_{K, L}}\right| \leq C(\alpha)\left|\frac{u_{L}-u_{K}}{d_{K, L}}\right|\left(\left|u_{L}\right|^{\alpha-1}+\left|u_{K}\right|^{\alpha-1}\right),
$$

using the Hölder inequality on the above product and using hypothesis 9.19 to upper bound $\left|\sigma_{K, L}\right| d_{K, L}$ by $|K|$ and by $|L|$ (this replaces the mesh proportionality assumption made in [24]) we get the inequality

$$
\int_{\Omega}|u|^{\alpha 1^{*}} \leq C(\Omega, d, p, \omega, \Gamma, \operatorname{reg}(\mathcal{T}))\left\{\left(\left\|\nabla_{h} u\right\|_{L^{p}(\Omega)} \times\left(\int_{\Omega}|u|^{(\alpha-1) \frac{p}{p-1}}\right)^{\frac{p-1}{p}}\right)^{1^{*}}\right.
$$




$$
\left.+\left(\left\|\nabla_{h} u\right\|_{L^{p}(\Omega)}\right)^{\alpha 1^{*}}+\left(m_{u}\right)^{\alpha 1^{*}}\right\} .
$$

Because we have $\alpha=p$, there holds

$$
\alpha \leq \frac{p(d-1)}{d-p} .
$$

From 9.25 one easily deduces that there is a continuous embedding of $L^{(\alpha-1) \frac{p}{p-1}}(\Omega)$ into $L^{\alpha 1^{*}}(\Omega)$. Using the weighted Young inequality, we derive

$$
\int_{\Omega}|u|^{\alpha 1^{*}} \leq C(\Omega, d, p, \omega, \Gamma, \operatorname{reg}(\mathcal{T}))\left(\left(\left\|\nabla_{h} u\right\|_{L^{p}(\Omega)}\right)^{\alpha 1^{*}}+\left(m_{u}\right)^{\alpha 1^{*}}\right)+\frac{1}{2} \int_{\Omega}|u|^{\alpha 1^{*}} .
$$

This yields the desired inequality (9.21) with $q=p 1^{*}$.

In the second step, we bootstrap the obtained estimate using the same technique. Namely, we set $\alpha:=\min \left\{p 1^{*}, p^{*} / 1^{*}\right\}$. Notice that from the result of the previous step, the bound $(9.22)$ follows.

Using (9.24) and 9.25) once more, we get 9.21) either for $q=p^{*}$ (this would end the proof), of for $q=p\left(1^{*}\right)^{2}$ (in which case we set $\alpha=\min \left\{p\left(1^{*}\right)^{2}, p^{*} / 1^{*}\right\}$ and bootstrap the argument). Because $p\left(1^{*}\right)^{k}$ goes to $+\infty$ as $k \rightarrow+\infty$, in a finite number of steps (thus, keeping the constant $C$ finite) we get 9.21 for $q=p *$.

As a concluding remark, notice that the following more precise version of 9.21 , well known in the continuous case:

$$
\left\|u-m_{u}\right\|_{L^{q}(\Omega)} \leq C(\Omega, d, p, q, \omega, \Gamma, \operatorname{reg}(\mathcal{T}))\left\|\nabla_{h} u\right\|_{L^{p}(\Omega)}
$$

does not seem attainable with the above technique. Notice that Glitzky and Griepentrog 29] do prove the subcritical embeddings in the form (9.26), for $p=2$ and on Voronoï meshes.

\section{ACKNOWLEDGMENT}

This work was initiated at the University of Concepción and supported by the chilean FONDECYT program No. 7080187. The third author acknowledges financial support by the European Research Council through the Advanced Grant "Mathcard, Mathematical Modelling and Simulation of the Cardiovascular System", project ERC-2008-AdG 227058. Part of this work was done while the second and third authors visited the Department of Mathematics at the University of Franche-Comté, France, and they are grateful for the kind hospitality.

\section{REFERENCES}

[1] M. Afif and B. Amaziane, Convergence of finite volume schemes for a degenerate convection-diffusion equation arising in flows in porous media, Comput. Methods Appl. Mech. Eng. 191 (2002) 5265-5286.

[2] D. Alonso, F. Bartumeus and J. Catalan, Mutual interference between predators can give rise to Turing spatial patterns, Ecology 83 (2002) 28-34.

[3] V. Anaya, M. Bendahmane and M. Sepúlveda, A numerical analysis of a reaction-diffusion system modelling the dynamics of growth tumors, Math. Meth. Models Appl. Sci., to appear.

[4] B. Andreianov, M. Bendahmane and K.H. Karlsen, Finite volume schemes for doubly nonlinear degenerate parabolic equations, J. Hyp. Diff. Eq., to appear.

[5] B. Andreianov, M. Gutnic and P. Wittbold, Convergence of finite volume approximations for a nonlinear ellipticparabolic problem: a "continuous" approach, SIAM J. Numer. Anal. 42 (2004) 228-251.

[6] J.W. Barrett and J.F. Blowey, Finite element approximation of a nonlinear cross-diffusion population model, Numer. Math. 98 (2004) 195-221.

[7] M. Bendahmane, Analysis of a reaction-diffusion system modeling predator-prey with prey-taxis, Netw. Heter. Media 3-4 (2008) 863-879.

[8] M. Bendahmane, Mathematical analysis of a cross-diffusion system modeling the propagation of an epidemic disease, Preprint 2008-09, Depto. Ing. Mat., U. de Concepción, available from http://www.ing-mat.udec.cl/; submitted.

[9] M. Bendahmane, Weak and classical solutions to predator-prey system with cross-diffusion, submitted.

[10] M. Bendahmane, T. Lepoutre, A. Marrocco and B. Perthame, Conservative cross diffusions and pattern formation through relaxation, J. Math. Pure Appl. 92 (2009) 651-667. 
[11] C. Chainais-Hillairet and J. Droniou, Finite volume schemes for non-coercive elliptic problems with Neumann boundary conditions, IMA J. Numer. Anal., in press.

[12] J. Chattopadhyay and S. Chatterjee, Cross diffusional effect in a Lotka-Volterra competitive system, Nonlinear Phenom. Complex Syst. 4 (2001) 364.

[13] L. Chen and A. Jüngel, Analysis of a multi-dimensional parabolic population model with strong cross-diffusion, SIAM J. Math. Anal. 36 (2004) 301-322.

[14] L. Chen and A. Jüngel, Analysis of a parabolic cross-diffusion population model without self-diffusion, J. Diff. Eqns. 224 (2006) 39-59.

[15] R. Cherniha and L. Myroniuk, New exact solutions of a nonlinear cross-diffusion system, J. Phys. A: Math. Theor. 41 (2008) 395-204.

[16] J.M. Chung and E. Peacock-López, Cross-diffusion in the Templator model of chemical self-replication, Phy. Lett. A 371 (2007) 41-47.

[17] Y. Coudière, T. Gallouët and R. Herbin, Discrete Sobolev inequalities and $L^{p}$ error estimates for finite volume solutions of convection diffusion equations, M2AN Math. Model. Numer. Anal. 35 (2001) 767-778.

[18] K. Domelevo and P. Omnès, A finite volume method for the Laplace equation on almost arbitrary two-dimensional grids, M2AN Math. Model. Numer. Anal. 39 (2005) 1203-1249.

[19] L.C. Evans, Partial differential equations, Volume 19 of Graduate Studies in Mathematics (American Mathematical Society, Providence, RI, 1998).

[20] R. Eymard, T. Gallouët and R. Herbin, Finite Volume Methods. In: P.G. Ciarlet, J.L. Lions (eds.), Handbook of Numerical Analysis, vol. VII, North-Holland, Amsterdam, (2000) pp. 713-1020.

[21] R. Eymard, T. Gallouet and R. Herbin. Finite volume approximation of elliptic problems and convergence of an approximate gradient. Appl. Num. Math., 37 (2001) 31-53.

[22] R. Eymard, T. Gallouët, R. Herbin, M. Gutnic and D. Hilhorst, Approximation by the finite volume method of an elliptic-parabolic equation arising in environmental studies, Math. Models Methods Appl. Sci. 11(9) (2001) $1505-1528$

[23] R. Eymard, T. Gallouët, R. Herbin and A. Michel, Convergence of a finite volume scheme for nonlinear degenerate parabolic equations, Numer. Math. 92 (2002) 41-82.

[24] R. Eymard, T. Gallouët and R. Herbin, Discretisation of heterogeneous and anisotropic diffusion problems on general non-conforming meshes. SUSHI: a scheme using stabilisation and hybrid interfaces, IMA J. Numer. Anal., in press.

[25] F. Filbet, A finite volume scheme for the Patlak-Keller-Segel chemotaxis model, Numer. Math. 104 (2006) 457-488.

[26] G. Galiano, M.L. Garzón and A. Jüngel, Semi-discretization and numerical convergence of a nonlinear cross-diffusion population model, Numer. Math. 93 (2003) 655-673.

[27] G. Galiano, M.L. Garzón and A. Jüngel, Analysis and numerical solution of a nonlinear cross-diffusion system arising in population dynamics, Rev. R. Acad. Cien. Serie A. Mat. 95 (2001) 281-295.

[28] G. Gambino, M.C. Lombardo and M. Sammartino, A velocity-diffusion method for a Lotka-Volterra system with nonlinear cross and self-diffusion, Appl. Num. Math. 59 (2009) 1059-1074.

[29] A. Glitzky and J.A. Griepentrog, Discrete Sobolev-Poincaré inequalities for Voronoï finite volume approximations, Preprint 2009, WIAS Berlin.

[30] A. Handlovičová, K. Mikula and F. Sgallari, Semi-implicit complementary volume scheme for solving level set like equations in image processing and curve evolution, Numer. Math. 93 (2003) 675-695.

[31] F. Hermeline, A finite volume method for approximating 3D diffusion operators on general meshes, Comput. Meth. Appl. Mech. Engrg. 228 (2009) 5763-5786.

[32] D. Horstmann, Remarks on some Lotka-Volterra type cross-diffusion models, Nonl. Anal.: Real World Appl. 8 (2007) 90-117.

[33] S. Kovács, Turing bifurcation in a system with cross diffusion, Nonl. anal. 59 (2004) 567-581.

[34] S.N. Kruzhkov. Results on the nature of the continuity of solutions of parabolic equations and some of their applications. Mat. Zametki 6(1) (1969) 97-108; English tr. in Math. Notes 6(1) (1969) 517-523.

[35] S.A. Levin and L.A. Segel, Pattern generation in space and aspect, SIAM Rev. 27 (1985) 45-67.

[36] J.-L. Lions, Quelques Méthodes de Résolution Des Problèmes Aux Limites Non Linéaires (Dunod, Paris, 1969).

[37] Y. Lou, W. -M. Ni and S. Yotsutani, On a limiting system in the Lotka- Volterra competition with cross-diffusion, Discr. Cont. Dynam. Sys. 10 (2004) 435-458.

[38] H. Matano and M. Mimura, Pattern formation in competition-diffusion systems in nonconvex domains, Publ. RIMS, Kyoto Univ. 19 (1983) 1049-1079.

[39] M. Mimura and K. Kawasaki, Spatial segregation in competitive interaction-diffusion equations, J. Math. Bio. 9 (1980) 49-64.

[40] J.D. Murray, Mathematical Biology II: Spatial Models and Biomedical Applications, Third Edition (Springer-Verlag, New York, 2003).

[41] A. Okubo, P.K. Maini, M.H. Williamson and J.D. Murray, On the spatial spread of the grey squirrel in Britain, Proc. R. Soc. London B 238 (1989) 113-125. 
[42] C.V. Pao, Strongly coupled elliptic systems and applications to Lotka-Volterra models with cross-diffusion, Nonl. Anal. 60 (2005) 1197-1217.

[43] M. Rodrigo and M. Mimura, Exact solutions of a competition-diffusion system, Hiroshima Math. J. 30 (2000) $257-270$.

[44] N. Shigesada and K. Kawasaki, Biological Invasions: Theory and Practice (Oxford University Press, 1997).

[45] N. Shigesada, K. Kawasaki and E. Teramoto, Spatial segregation of interacting species, J. Theor. Bio. 79 (1979) $83-99$.

[46] C. Tian, Z. Lin and M. Pedersen, Instability induced by cross-diffusion in reaction-diffusion systems, Nonl. Anal.: Real World Appl. 11 (2010) 1036-1045.

[47] Z. Wen and S. Fu, Global solutions to a class of multi-species reaction-diffusion systems with cross-diffusions arising in population dynamics, J. Comp. Appl. Math. 230 (2009) 34-43. 